\title{
Multiscale Tow-Phase Flow Modeling of Sheet and Cloud Cavitation
}

\author{
Chao-Tsung Hsiao ${ }^{\mathrm{a}}{ }_{*}$, Jingsen Ma ${ }^{\mathrm{a}}$, Georges L. Chahine ${ }^{\mathrm{a}}$ and \\ a'DYNAFLOW, INC., 10621-J Iron Bridge Road, Jessup, MD, USA
}

\begin{abstract}
A multiscale two-phase flow model based on an Eulerian/Lagrangian coupled approach is applied to capture the sheet cavitation formation, development, unsteady breakup, and bubble cloud shedding on a hydrofoil. No assumptions are needed on mass transfer. Instead natural free field nuclei and solid boundary nucleation are modelled and enable capture of the sheet and cloud dynamics. The multiscale model includes a micro-scale model for tracking the bubbles, a macroscale model for describing large cavity dynamics and a transition scheme to bridge the micro and macro scales. With this multiscale model small nuclei are seen to grow into large bubbles, which eventually merge to form a large scale sheet cavity. A reentrant jet forms under the sheet cavity, travels upstream, and breaks the cavity, resulting in the emission of high pressure peaks as the broken pockets shrink and collapse while travelling downstream. The results for a 2D NACA0015 foil are in good agreement with published experimental measurements in terms of sheet cavity lengths and shedding frequencies. Sensitivity assessment of the model parameters and 3D effects on the predicted major cavity dynamics are also discussed.
\end{abstract}

\section{INTRODUCTION}

Various types of cavitation occur over a propeller blade. These include traveling bubble cavitation, tip vortex cavitation, sheet cavitation, cloud cavitation, supercavitation, and combinations of the

* Corresponding author.

Emails: ctsung@dynaflow-inc.com; jingsen@dynaflow-inc.com; glchahine@dynaflow-inc.com

Phone: 301-604-3688 Fax: 301-604-3689

(C) 2016. This manuscript version is made available under the Elsevier user license

http://www.elsevier.com/open-access/userlicense/1.0/ 
above. Among these, the transition from sheet to cloud cavitation is recognized as the most erosive and is quite difficult to accurately model. The sheet cavity starts near the leading edge, effectuates large amplitude length/volume oscillations, and breaks up periodically releasing clouds of bubbles and cavitating vortical structures, which collapse violently generating high local pressures on the blade. Accurate simulation and modeling of the physics involved is challenging since the problems of sheet cavity dynamics, cloud cavity dynamics, and sheet-to-cloud transition involve length and time scales of many different orders of magnitude.

Experimental observations have indicated weak or little dependence of the sheet cavity inception and dynamics on the nuclei distribution in the test facility, which has led many numerical modelers to believe that sheet cavitation is not connected with bubble dynamics. Actually, over the years, several researchers (e.g. Kodama 1981, Kuiper 2010) have observed that the presence of free field nuclei affected significantly the repeatability of the experimental results. In experiments where nuclei count was very low the value of the incipient cavitation number showed large scatter, whereas in water rich in nuclei the results were more repeatable and were not affected by further addition of nuclei. One potential explanation to the above observations is that nucleation from the solid surface of the blades could play a much more important role than the free field nuclei, and this may have been overlooked in previous studies of sheet cavitation even though bubble nucleation has been extensively investigated (e.g. Harvey et al. 1944, Yount 1979, Mørch 2009).

Conventional numerical approaches for modeling sheet cavities assume that the cavity is filled with vapor at the liquid vapor pressure and thus arbitrarily define as a liquid-cavity interface the liquid iso-pressure surface with the vapor pressure value. The flow solution in the liquid is obtained using either a Navier-Stokes solver (Deshpande et al. 1993, Chen and Heister 1994, Hirschi et al 1998) or a potential flow solver using a boundary element method (Kinnas and Fine 1993, Chahine and Hsiao 2000, Varghese et al. 2005). Since the developed sheet cavity is very unsteady and dynamic, numerical methods requiring a moving grid to resolve the flow field are accurate but encounter numerical difficulties to describe folding or breaking up interfaces. Other approaches have thus been developed to avoid using moving grids to track the liquid-gas interface. They are mainly in two categories: Volume of Fluid methods (VoF) (Merkle et al. 1998, Kunz et al. 2000, Yuan et al. 2001, Singhal et al. 2002) and Level-Set Methods (LSM) (Kawamura and Sakoda 2003, Dabiri et al. 2007, 2008, Kinzel et al 2009). While both interface capturing methods solve the Navier-Stokes equations using a fixed computational grid, the liquid/gas interface is captured differently. In the VoF method, an advection equation for the volume fraction of the vapor is solved and the two-phase medium density is computed knowing the volume fraction of each phase. This method does not have a sharp interface with boundary conditions but applies a mass transfer model 
between the liquid and the cavity to model liquid/vapor exchange at the interface using 'calibration' parameters to match experimental results. In the level set method, the interface location is determined by solving an advection equation for the level set function with the interface represented by the zero level (or iso-surface) of the level-set function. This method provides a sharp interface on which boundary conditions similar to those used in an interface tracking scheme (e.g. a constant pressure value and zero shear stress) can be imposed.

Interface capturing approaches have been successfully applied to describe the dynamic evolution of a cavitation sheet and its breakup but not to the dispersed bubble phase and to the resolution of the transition from sheet to bubbles since this would require an extremely fine spatial resolution relative to bubble size (Fedkiw et al. 1999, Osher and Fedkiw 2001). Resolving sheet cavity together with the microbubbles involves characteristic lengths of different orders of magnitude and using the smallest scale requires computational resources not currently available.

Instead of resolving all individual bubbles, continuum-based Eulerian approaches ( Biesheuvel and Van Wijngaarden 1984, Zhang and Prosperetti 1994 a,b, Druzhinin and Elghobashi 1998, 1999, 2001, Ma et al. 2011, 2012) assuming the bubbly phase to be a continuum are typically used for bubbles much smaller than the characteristic lengths associated with the motion of the overall mixture. In this case, the precise location and properties of individual bubbles are not directly apparent at the global mixture flow scale. As a result, this approach is not able to address appropriately the bubble dynamics and interactions which are important in cavitation and more so in cloud cavitation.

Approaches combining both continuum and discrete bubbles have been proposed (Chahine and Duraiswami 1992, Chahine, 2009, Balachandar and Eaton 2010, Raju et al. 2011, Shams et al. 2011, Hsiao et al. 2013). In these approaches the carrier fluid is modeled using standard continuum fluid methods (Eulerian approach) and dispersed bubbles are tracked directly (Lagrangian approach). The interaction between the two phases is achieved through a locally distributed force field imposed in the momentum equation (Ferrante and Elghobashi 2004, Xu et al. 2002) or through the inclusion of local density variation due to bubble motion and dynamics (Chahine, 2009, Raju et al. 2011, Hsiao et al. 2013, Ma et al. 2014)..

We have recently developed a multiscale method, which aims at addressing all the various scales of the problem; notably a macro-scale at the order of the blade length, a micro-scale for the bubble nuclei, an intermediary scale for the bubble clouds, and transition and coupling procedures between these scales. To include sheet cavitation inception in the study, we pursue along the same concept that we have pioneered to study travelling bubble or tip vortex cavitation inception (Chahine, 2004, Hsiao and Chahine 2004, 2008, Chahine, 2009). This starts from the precept that 
water always contains microscopic bubble nuclei and solid liquid interfaces do not have perfect contact and thus entrap nanoscopic or microscopic gas pockets, which are a source of nucleation (freeing of nuclei into the liquid) when appropriate local conditions occur. Cavitation inception of all types is initiated through explosive growth of these nuclei.

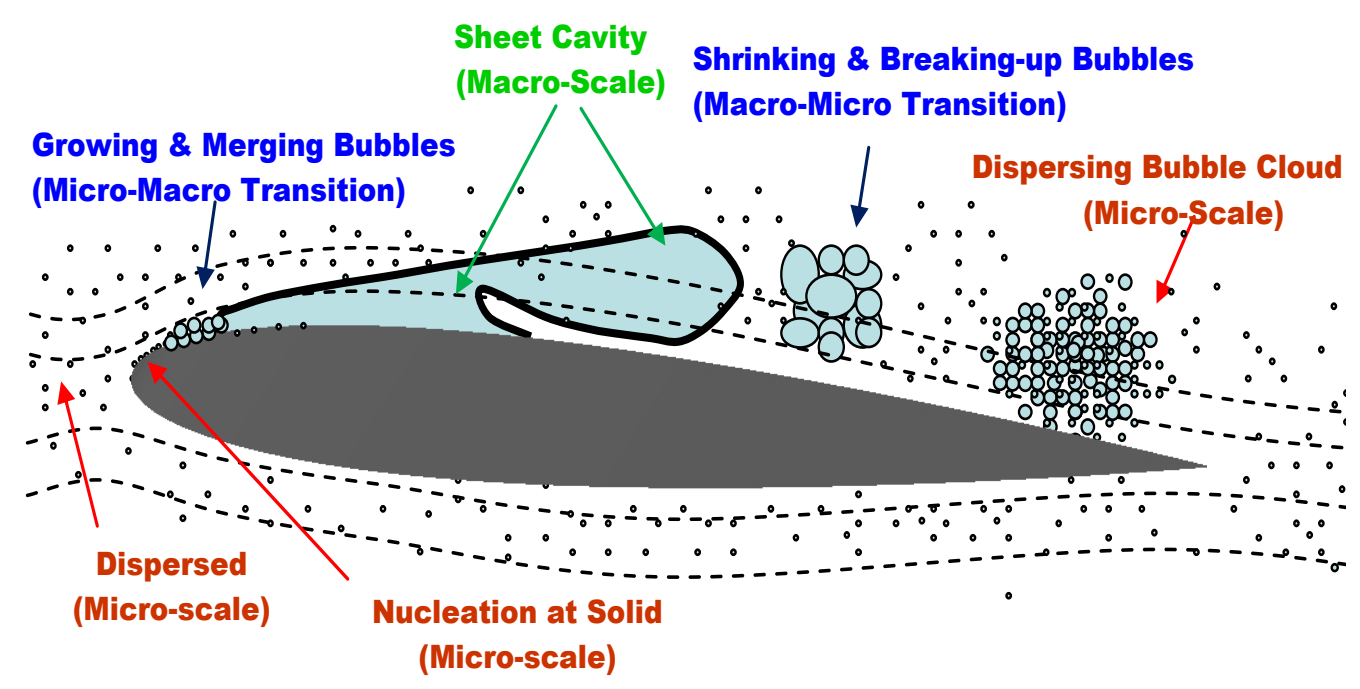

Figure 1: Overview of the various scales involved in the problem of cavitation on a foil and sketch of the modeling strategy.

\section{NUMERICAL MODEL}

The sketch in Figure 1 presents an overview of the multiscale model developed in this study. This model aims at addressing the physics at the various scales involved in sheet and cloud cavitation:

- At the micro-scale, transport of nuclei and microbubbles, nucleation from solid surfaces, and corresponding bubble dynamics are considered. The model addresses dispersed pre-existing nuclei in the liquid, nuclei originating from solid boundaries, and micro-bubbles resulting from disintegration of sheet and cloud cavities or bubbles breaking up from vaporous/gaseous cavities. At this scale, the model tracks the bubbles in a Lagrangian fashion.

- At the macro-scale, a two-phase continuum-based flow is solved on an Eulerian grid. At this scale gas/liquid interfaces of large bubbles, air pockets, and cavities are directly discretized and resolved through tracking the gas-liquid interfaces with a Level Set method.

- Inter-scale transition schemes are used to bridge micro and macro scales as bubbles grow or merge to form large cavities, or as bubbles shrink or break up from a large cavity. At this scale, 
model transition between micro-scale and macro-scale and vice-versa is realized by using information from both the Discrete Singularity Model (DSM) and the Level Set method (LSM). An Eulerian/Lagrangian coupled two-phase flow model is used. The Eulerian model addresses the continuum-based two-phase model and is coupled with a discrete bubble model tracking bubble motion using a Lagrangian approach.

\section{Eulerian Continuum-Based Two-Phase Model}

The two-phase medium continuum model uses a viscous flow solver to solve the Navier-Stokes equations and satisfy the continuity and momentum equations:

$$
\begin{gathered}
\frac{\partial \rho_{m}}{\partial t}+\nabla \cdot \rho_{m} \mathbf{u}=0 \\
\rho_{m} \frac{D \mathbf{u}}{D t}=-\nabla p+\nabla\left\{\tau_{i j}-\frac{2}{3} \mu_{m} \nabla \cdot \mathbf{u} \quad \delta_{i j}\right\}, \\
\tau_{i j}=\mu_{m}\left(\frac{\partial u_{i}}{\partial x_{j}}+\frac{\partial u_{j}}{\partial x_{i}}\right)
\end{gathered}
$$

where the subscript $m$ refers to the mixture properties. $\mathbf{u}$ is the mixture velocity, $p$ is the pressure, and $\delta_{i j}$ is the Kronecker delta. The mixture density, $\rho_{m}$, and the mixture viscosity, $\mu_{m}$, can be expressed as functions of the void volume fraction $\alpha$ :

$$
\rho_{m}=1-\alpha \rho_{l}+\alpha \rho_{g}, \quad \mu_{m}=1-\alpha \mu_{l}+\alpha \mu_{g},
$$

where the subscript $l$ refers to the liquid and the subscript $g$ refers to the gas.

The equivalent medium has a time and space dependent density since the void fraction $\alpha$ varies in both space and time. This makes the overall flow field problem similar to a compressible flow problem. In our approach, which couples the continuum medium with the discrete bubbles, the mixture density is not an explicit function of the pressure through an equation of state. Instead, tracking the bubbles and knowing their concentration provides $\alpha$ and $\rho_{m}$ as functions of space and time. This is achieved by using a Gaussian distribution scheme which smoothly "spread" each bubble's volume over neighboring cells within a selected radial distance while conserving the total bubble volumes (Ma et al., 2014). Specifically, in a fluid cell $i$, the void fraction is computed using:

$$
\alpha_{i}=\sum_{j=1}^{N_{i}} \frac{f_{i, j} V_{j}^{b}}{\sum_{k}^{N_{\text {cels }}} f_{k, j} V_{k}^{\text {cell }}},
$$


where $V_{j}^{b}$ and $V_{k}^{\text {cell }}$ are the volumes of a bubble $\mathrm{j}$ and cell k respectively, $N_{i}$ is the number of bubbles which are influencing cell $i . N_{\text {cells }}$ is the total number of cells "influenced" by a bubble $j . f_{i, j}$ is the weight of the contribution of bubble $j$ to cell $i$ and is determined by the Gaussian distribution function. This scheme has been found to significantly increase numerical stability and to enable the handling of high-void bubbly flow simulations.

The system of equations is closed by an artificial compressibility method (Chorin, 1967) in which a pseudo-time derivative of the pressure multiplied by an artificial-compressibility factor, $\beta$, is added to the continuity equation as

$$
\frac{1}{\beta} \frac{\partial p}{\partial \tau}+\frac{\partial \rho_{m}}{\partial t}+\nabla \cdot \rho_{m} \mathbf{u}=0
$$

As a consequence, a hyperbolic system of equations is formed and can be solved using a time marching scheme. This method can be marched in pseudo-time to reach a steady-state solution. To obtain a time-dependent solution, a Newton iterative procedure is performed at each physical time step in order to satisfy the continuity equation.

The Navier-Stokes solver uses a finite volume formulation. First-order Euler implicit differencing is applied to the time derivatives. The spatial differencing of the convective terms uses the flux-difference splitting scheme based on Roe's method (Roe 1981) and van Leer's MUSCL method (van Leer 1979) for obtaining the first-order and the third-order fluxes, respectively. A second-order central differencing is used for the viscous terms which are simplified using the thinlayer approximation. The flux Jacobians required in an implicit scheme are obtained numerically. The resulting system of algebraic equations is solved using the Discretized Newton Relaxation method (Vanden, and Whitfield 1995) in which symmetric block Gauss-Seidel sub-iterations are performed before the solution is updated at each Newton iteration.

\section{Level-Set Approach}

In order to enable the multiscale code to simulate large free surface deformations such as folding and breakup in the sheet cavity problem, a Level-Set method is used. A smooth function $\varphi$ $(x, y, z, t)$, whose zero level coincides with the liquid/gas interface when the level set is introduced, is defined in the whole physical domain (i.e. in both liquid and gas phases) as the signed distance $d(x, y, z)$ from the interface:

$$
\varphi x, y, z, 0=d x, y, z
$$

This function is enforced to be a material surface at each time step using: 


$$
\frac{d \varphi}{d t}=\frac{\partial \varphi}{\partial t}+\mathbf{u} \nabla \varphi=0
$$

where $\mathbf{u}$ is the velocity of interface. Integration of Equation (7) does not ensure that the thickness of the interface region remains constant in space and time during the computations due to numerical diffusion and to distortion by the flow field. To avoid this problem, a new distance function is constructed by solving a "re-initialization equation" in pseudo time iterations (Sussman 1998):

$$
\frac{\partial \varphi}{\partial \tau}=S \varphi_{0}[1-|\nabla \varphi|]
$$

where $\tau$ is the pseudo time, $\varphi_{0}$ is the initial distribution of $\varphi$, and $S\left(\varphi_{0}\right)$ is a sign function, which is zero at the interface.

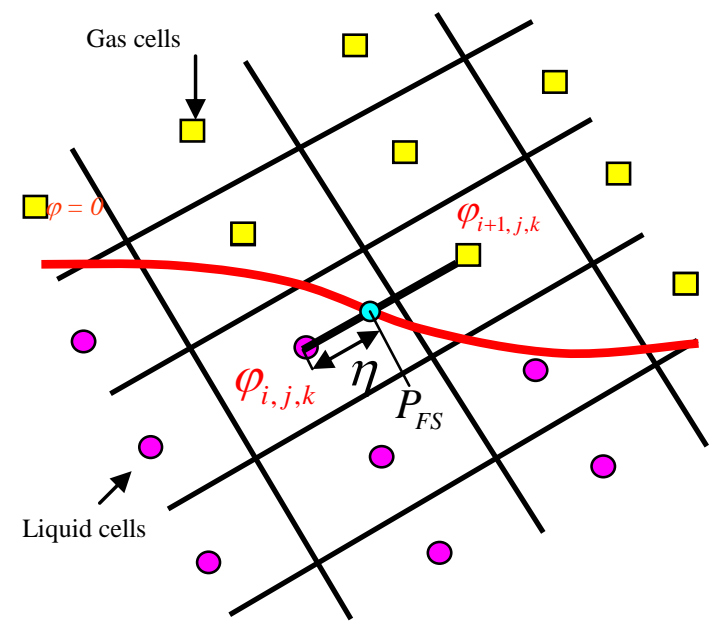

Figure 2: Cell location identification in the computational domain.

In a standard Level-Set approach liquid and gas phases are solved separately using Equations (7) and (8) after identifying to which phase a concerned cell belongs and applying a smoothed Heaviside function over the interface to smooth the fluid properties. Figure 2 illustrates the identification of the cells according to the zero level-set.

Instead of solving both phases, we have implemented a single phase level-set method using the Ghost Fluid Method (Fedkiw et al. 1999, Kang et al. 2000) to maintain a sharper interface. This method allows imposing the dynamics boundary conditions across the interface without use of smoothing functions. If the shear due to the air is neglected, the dynamic boundary conditions (balance of normal stresses and zero shear) can be written: 


$$
p=\frac{\mathbf{n} \bullet \boldsymbol{\tau} \bullet \mathbf{n}}{\rho_{l}}+\frac{g z}{\rho_{l}}+\frac{\gamma \kappa}{\rho_{l}}, \quad \mathbf{n} \cdot \boldsymbol{\tau} \bullet \mathbf{t}=0
$$

where $\tau$ is the stress tensor, $g$ is the acceleration of gravity, and $\gamma$ is the surface tension. $\kappa=\nabla \cdot \nabla \varphi|\nabla \varphi|$ is the surface curvature. $\mathbf{n}$ and $\mathbf{t}$ are the surface normal and tangential vectors, respectively.

In this approach one or more ghost cells are used to impose the boundary conditions when the interface cells are solved. The interface cells for each phase are identified when one of the following six inequalities is satisfied for two adjacent cells:

$$
\begin{aligned}
& \varphi_{i, j, k} \varphi_{i \pm 1, j, k}<0, \\
& \varphi_{i, j, k} \varphi_{i, j \pm 1, k}<0, \\
& \varphi_{i, j, k} \varphi_{i, j, k \pm 1}<0 .
\end{aligned}
$$

Although the Ghost Fluid Method can help maintain a sharp interface, additional computational cost is required for the cells in the ignored phase, e.g. air. To further reduce the computational cost we apply a single-phase level-set approach in which only the liquid phase of the fluid is solved, while the cells belonging to the gas phase are deactivated during the computations. To solve Equation (7) we use the velocity information from the gas cells which are near the interface. To obtain this information, we assume the following condition across the interface:

$$
\mathbf{n} \bullet \nabla \mathbf{u}=0, \quad \mathbf{n}=\nabla \varphi /|\nabla \varphi|
$$

which means there is no normal component of the velocity gradient at the interface. With this assumption we can extend the velocity from the liquid phase along the normal direction of the distance function to the gas phase by solving the pseudo time iteration equation:

$$
\frac{\partial \mathbf{u}}{\partial \tau}+\mathbf{n} \cdot \nabla \mathbf{u}=0
$$

\section{Lagrangian Discrete Bubble Model}

The Lagrangian discrete bubble model is based on the discrete singularity model which uses a Surface Average Pressure (SAP) approach (Hsiao et al., 2003, Chahine, 2004, Choi et al., 2004) to average fluid quantities along the bubble surface. This model has been shown to produce accurate results when compared to full 3D two-way interaction computations (Hsiao and Chahine, 2003). The averaging scheme allows one to consider only a spherical equivalent bubble and use a modified Rayleigh-Plesset equation to describe the bubble dynamics, 


$$
\rho_{l}\left(R \ddot{R}+\frac{3}{2} \dot{R}^{2}\right)=p_{v}+p_{g 0}\left(\frac{R_{0}}{R}\right)^{3 k}-p_{\text {enc }}-\frac{2 \gamma}{R}-\frac{4 \mu \dot{R}}{R}+\rho_{l} \frac{\left|\mathbf{u}_{s}\right|^{2}}{4}
$$

where the slip velocity, $\mathbf{u}_{s}=\mathbf{u}_{e n c}-\mathbf{u}_{b}$, is the difference between the encountered (SAP averaged) liquid velocity, $\mathbf{u}_{e n c}$, and the bubble translation velocity, $\mathbf{u}_{b} . R$ and $R_{0}$ are the bubble radii and time $t$ and $0, p_{v}$ is the liquid vapor pressure, $p_{\mathrm{g} 0}$ is the initial bubble gas pressure, $k$ is the polytropic compression constant, and $p_{e n c}$ is the average SAP pressure "seen" by the bubble during its travel. With the SAP model, $\mathbf{u}_{e n c}$ and $p_{e n c}$ are respectively the averages of the liquid velocities and pressures over the bubble surface.

The bubble trajectory is obtained from the following bubble motion equation:

$$
\begin{aligned}
\frac{d \mathbf{u}_{b}}{d t}= & \left(\frac{\rho_{l}}{\rho_{b}}\right)\left[\frac{3}{8 R} C_{D}\left|\mathbf{u}_{s}\right| \mathbf{u}_{s}+\frac{1}{2}\left(\frac{d \mathbf{u}_{e n c}}{d t}-\frac{d \mathbf{u}_{b}}{d t}\right)+\right. \\
& \left.\frac{3 \dot{R}}{2 R} \mathbf{u}_{s}-\frac{\nabla p}{\rho_{l}}+\frac{\left(\rho_{b}-\rho_{l}\right)}{\rho_{l}} g+\frac{3 C_{L}}{4 \pi} \frac{\sqrt{\nu}}{R} \frac{\mathbf{u}_{s} \times \mathbf{\Omega}}{\sqrt{|\boldsymbol{\Omega}|}}\right],
\end{aligned}
$$

where $\rho_{b}$ is the density of the bubble, $C_{D}$ is the drag coefficient given by an empirical equation, e.g. from Haberman and Morton (1953), $C_{L}$ is the lift coefficient and $\Omega$ is the vorticity vector. The $1^{\text {st }}$ right hand side term is a drag force. The $2^{\text {nd }}$ and $3^{\text {rd }}$ terms account for the added mass. The $4^{\text {th }}$ term accounts for the presence of a pressure gradient, while the $5^{\text {th }}$ term accounts for gravity and the $6^{\text {th }}$ term is a lift force (Saffman 1965).

\section{Transition Model between Micro- and Macro-Scale}

The transition between micro- and macro-scale models includes two scenarios:

a. transition from collected coalescing microbubbles into relatively large two-phase cavities,

b. transition from a large cavity into a set of micro-scale dispersed bubbles.

In the first scenario a criterion based on bubble / cell relative size is set to "activate" bubbles for computation of a level set function:

$$
R \geq \max \left(R_{t h r}, m_{t h r} \Delta L\right),
$$

where $R_{t h r}$ is a threshold bubble radius, $\Delta L$ is the size of the local grid which hosts the bubble, and $m_{t h r}$ is a threshold grid factor. Therefore a bubble interface is switched to be represented by the zero level set when the bubble radius exceeds a threshold value and becomes larger than a multiple of the local grid size. 
At the beginning of the simulation where no cavity exists, all cells have the same level set value, $\varphi_{L S 0}$, fixed to be a very large negative value. When bubbles are detected to satisfy (15), the level set value of each cell in the computation domain is modified based on the distance from each cell center to the bubbles' surfaces, using the following equation:

$$
\varphi_{i}=\min \left(\varphi_{L S 0}, \varphi_{b, j}\right) ; j=1, N_{i}
$$

where $\varphi_{b, j}$ is the local distance function computed based on the surface of bubble $j$, and $N_{i}$ is the number of bubbles which are "activated" around cell $i$.

This scheme allows multiple bubbles to merge together into a large cavity, or isolated bubbles to coalesce with a previously defined large cavity.

In the second scenario, we use the zero level-set to identify the gas/liquid interface of the cavity. The volume of the cavity is tracked at each time step to determine when the cavity collapse occurs. As the cavity collapses, surface instabilities and cavity disintegration are accounted for using empirical criteria based on experimental observations. An amount of micro-bubbles of the same volume is initiated around the cavity to replace volume loss between time steps. The procedure is similar to that used to simulate bubble entrainment in breaking waves. Micro-bubbles are entrained into the liquid once the local normal velocity pointing towards the liquid exceeds the speed of the gas/liquid interface. This leads to a simple expression for the location and rate of bubble generation that is proportional to the liquid's local turbulent kinetic energy times the gradient of the liquid velocity in the liquid normal direction. The procedure is similar to that in Ma et al., 2011 and Hsiao et al., 2013.

\section{Nucleation Model}

The simulations presented below use free field nuclei as measured experimentally by many researchers such as (Medwin, H., 1977, M. L. Billet, 1985 Franklin, R.E., 1992, Wu, et al., 2010). In our modeling the bubbles are distributed randomly in the liquid following the measured bubble size distribution, as more detailed in our previous work in (Chahine, 2004, 2009, Hsiao et al. 2013). Another important source of nuclei for the present problem is nuclei periodically released from the blade surface. Boundary nucleation is easily observed in conditions where gas diffusion (beer or champagne glass) and/or boiling are dominant; bubbles grow out of distributed fixed spots. This phenomenon has been studied extensively in the past. Harvey et al. (1944) suggested that gas pockets, trapped in hydrophobic conical cracks and crevices of solid surfaces, act as cavitation nuclei. More recently Mørch (2009) investigated this effect in more depth and suggested that a skin model similar to that proposed by Yount (1979) for dispersed nuclei can also be used to describe 
the wall gas bubbles because solid surfaces submerged in a liquid tend to absorb amphiphilic molecules (molecules having a polar water-soluble group attached to a nonpolar water-insoluble hydrocarbon chain) and capture nanoscopic air pockets. One can expect that clusters of such molecules form weak spots, which are the source for bubble nucleation from the solid surface when low pressure conditions are achieved. Briggs (2004), for instance, found that scrupulous cleaning was decisive for obtaining a high tensile strength of water, and that contamination of interfaces is a primary factor in cavitation. Atchley and Prosperetti (1989) modeled nucleation out of crevices with liquid pressure drop. They identified unstable motion of the liquid-solid-gas contact line in the crevice and unstable growth of the nucleus volume due to loss of mechanical stability (force balance). Specifically, they related the threshold pressure to crevice aperture, surface tension, and gas concentration etc.

Based on existing experimental observations and theoretical studies the present work used a nucleation model with the following characteristics parameters:

- $P_{t h r}$ : a nucleation pressure threshold,

- $N_{s:}$ a number density of nucleation sites per unit area,

- $f_{n}$ : a nucleation time emission rate, function of the local flow condition,

- $R_{0}$ : initial nuclei size, function of the local flow condition.

In this nucleation model, at each time step, nuclei are released from a blade boundary cell when the pressure at the cell center drops below $P_{t h r}$. The cell of surface area $\Delta A$, then releases $N$ nuclei during the time interval $\Delta t$, obtained as follows:

$$
N=N_{s} f_{n} \Delta t \Delta A
$$

Since the Lagrangian solver (bubble tracking) usually requires time steps much smaller than the Eulerian flow solver, the $N$ nuclei can be released randomly in space from the area $\triangle A$ and in time during the Eulerian time step, $\Delta t$.

It is expected that the above parameters are also functions of surface roughness, $S$, temperature, $T$, and other physico-chemical parameters not explicitly considered here. In this study, we use this simplified nucleation model to investigate whether it enables with the above described overall multiscale approach to recover the main characteristics of sheet cavitation.

\section{SHEET CAVITATION ON 2D HYDROFOIL}

\section{Case Setup}

We study first the development of a sheet cavity over a 2D NACA0015 hydrofoil to study the sensitivity of the solution to the values of the parameters used in the numerical model. An $\mathrm{H}-\mathrm{H}$ 
type grid is generated with $181 \times 101$ grid points in the stream wise and normal direction, respectively. Only 2 grid points are used along the spanwise direction to enforce twodimensionality. 101 grid points are used on each side of the hydrofoil surface and non-slip wall boundary conditions are imposed there. The grid is subdivided into 12 blocks for a computational domain which extends 3.0 chord-length upstream and 5.0 chord-lengthdownstream. The grid also extends 2.0 chord-length vertically away from the foil surface where far-field boundary conditions are applied. The grid is designed to provide high clustering around the hydrofoil surface as shown in Figure 3. In particular, the first grid above the hydrofoil surface is located within $y^{+}<1$ in order to properly capture the boundary layer and apply turbulence modeling. Grid resolution was determined following grid convergence studies in previous work.

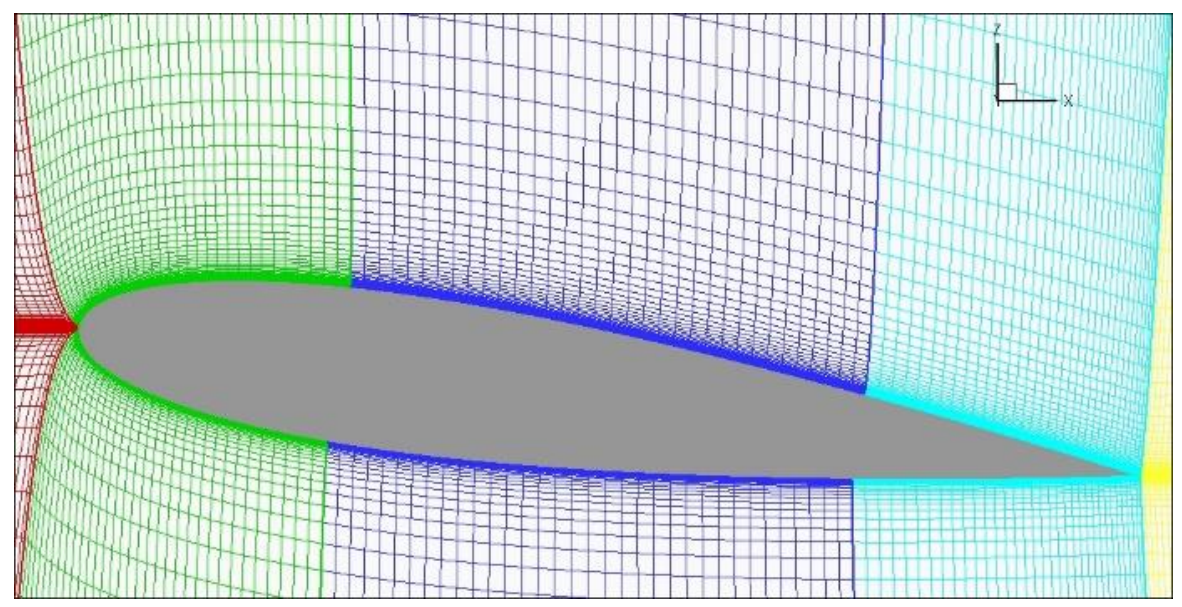

Figure 3: Zoomed-in view of the multi-block grid used in this study for the simulation of sheet cavitation on a NACA0015 hydrofoil. 


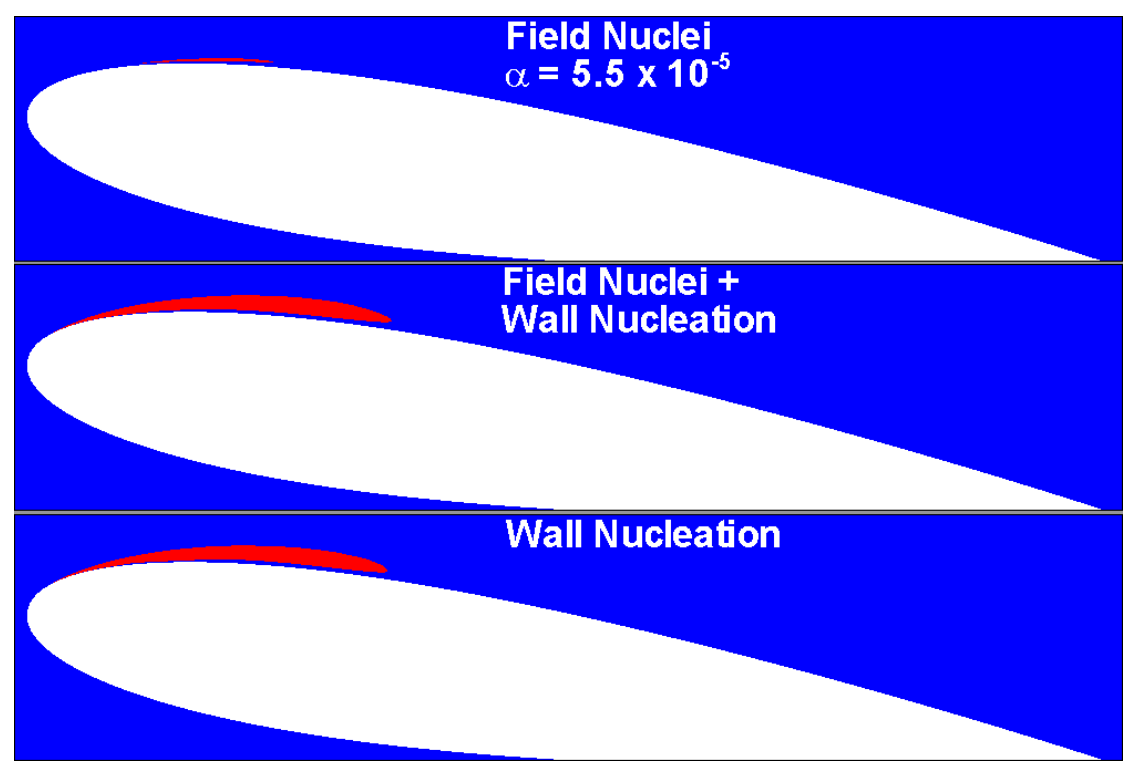

Figure 4: Importance of the inclusion of wall nucleation on the proper modeling of the sheet cavity using the Eulerian-Lagrangian and level set methods. Field nuclei alone do not reach the wall or grow enough to fill the cavity. $\sigma=1.65, \alpha=8^{\circ}, U_{\infty}=10 \mathrm{~m} / \mathrm{s}, L=0.12 \mathrm{~m}, N_{s}=224 \mathrm{~cm}^{-2}, F_{n}=22 \mathrm{kHz}$.

In this section, the foil is at an angle of attack of $8^{\circ}$ and $10^{\circ}$, its chord length is $0.12 \mathrm{~m}$, and the incoming flow from far-field upstream has a mean velocity of $10 \mathrm{~m} / \mathrm{s}$, resulting in a Reynolds number of $1.2 \times 10^{6}$. The nuclei are dispersed in the bulk of the liquid and are also released from the surface of the foil according to the solid surface nucleation model. The filed nuclei have radii ranging from $20 \mu \mathrm{m}$ to $60 \mu \mathrm{m}$, and correspond to an average initial void fraction of $5.5 \times 10^{-5}$.

Nucleation from the solid wall is initiated when the local pressure drops below a critical value, here selected to be the vapor pressure. The initial size of the nuclei emitted is selected to be $10 \mu \mathrm{m}$ and the number of bubbles is determined by Equation (17) with the number of nucleation sites per unit area selected to be $N_{s}=224 / \mathrm{cm}^{2}$ and the nucleation frequency selected to be $f_{n}=22 \mathrm{kHz}$. The microbubbles become a cavity described by a Level Set surface when they grow larger than the size of the local grid and exceed $350 \mu \mathrm{m}$ ( $\sim 10$ times the median initial nuclei size). The effects of these selected values of the parameters on the solution are investigated below.

Comparison of the numerical results with experimental observations are very satisfactory, as described in more detail in the next section, only when wall nucleation is taken into account. This is illustrated in Figure 4, which clearly shows a major difference between time average cavity sizes when wall nucleation is included or when it is ignored. Accounting for field nuclei only is unable to capture the sheet length properly unless the void fraction is relatively high (above $10^{-3}$ ). This is very much in agreement with the experimental observations. Including the wall nucleation alone is 
capable of capturing the sheet length but is insufficient to capture properly the events behind the cavity closure such as the development of cloud cavities and cavitating vortical structures.

\section{Dynamics of the Sheet Cavity}

A time sequence of sheet cavity shape oscillation and cloud cavitation shedding for one time cycle is shown in Figure 5 for $\sigma=1.65$. Nucleation initiates at the hydrofoil surface near the leading edge where the pressure drops below the selected nucleation threshold, $p_{t h r}=p_{v}$. Initially, bubble nuclei from the bulk of the liquid being convected downstream, as well as nuclei released from the solid surface, grow, coalesce and form a discrete small cavity described with a level set function. Later, free field nuclei have difficulty to enter in the cavity just move downward, while wall nucleation keeps feeding vapor and gas into the sheet cavity which grows to become a fully developed sheet cavity.

At the back of the cavity a stagnation flow forms (Shen and Dimotakis 1989) as shown in the zoomed view in Figure 6. The reverse flow results in a re-entrant jet, which moves upstream under the sheet cavity and ultimately contributes to breaking the cavity into two. The shed cavity collapses as it travels downstream generating high pressures. At the same time a new sheet cavity forms and expands, and the cycle repeats.

This periodic shedding phenomenon can be further quantified by analyzing the time histories of the instantaneous pressures and stream-wise velocities in the flow. For example Figure 7 shows the pressures and velocities monitored at the first grid point in the liquid on the suction side of the solid surface at the location $x=0.4 \mathrm{~L}$ downstream of the leading edge. This is at about the same location as the maximal extent of the trailing edge of the fully developed sheet cavity. This location alternatively experiences positive and negative stream-wise velocities with magnitudes as high as the incoming flow, $U_{\infty}$. The time interval between consecutive negative velocity maxima is repeatable and has a value close to $1.15 L / U_{\infty}$. The corresponding oscillation frequency is about $0.86 U_{\infty} / L$, which matches very well with the experimentally measured shedding frequency for the same cavitation number (Berntsen et al. 2001). 


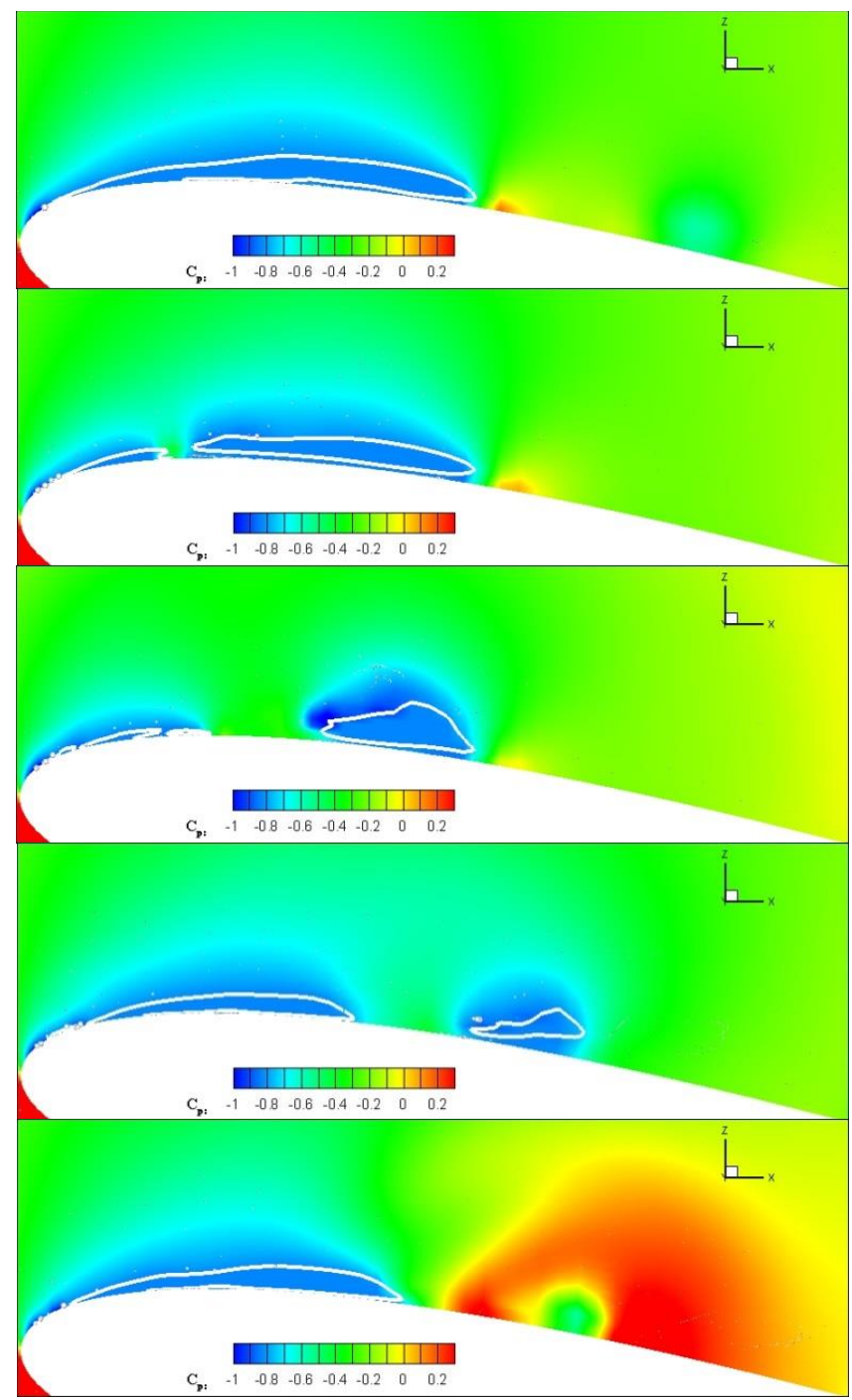

Figure 5: Time sequence of sheet cavitation formation and cloud shedding with pressure contours. $\left(\sigma=1.65, \alpha=8^{\circ}, U_{\infty}=10 \mathrm{~m} / \mathrm{s}, L=0.12 \mathrm{~m}\right)$. The white dots are discrete bubbles and the white lines are the zero level-set lines denoting the liquid-vapor interface.

Figure 7 also shows that each cycle of flow velocity oscillations coincides with the alternation of peaks and minimum pressure values with the same oscillation frequency. This illustrates a strong correlation between reentrant jet initiation and the development of pressure fluctuations during each cycle of the sheet cavitation. 

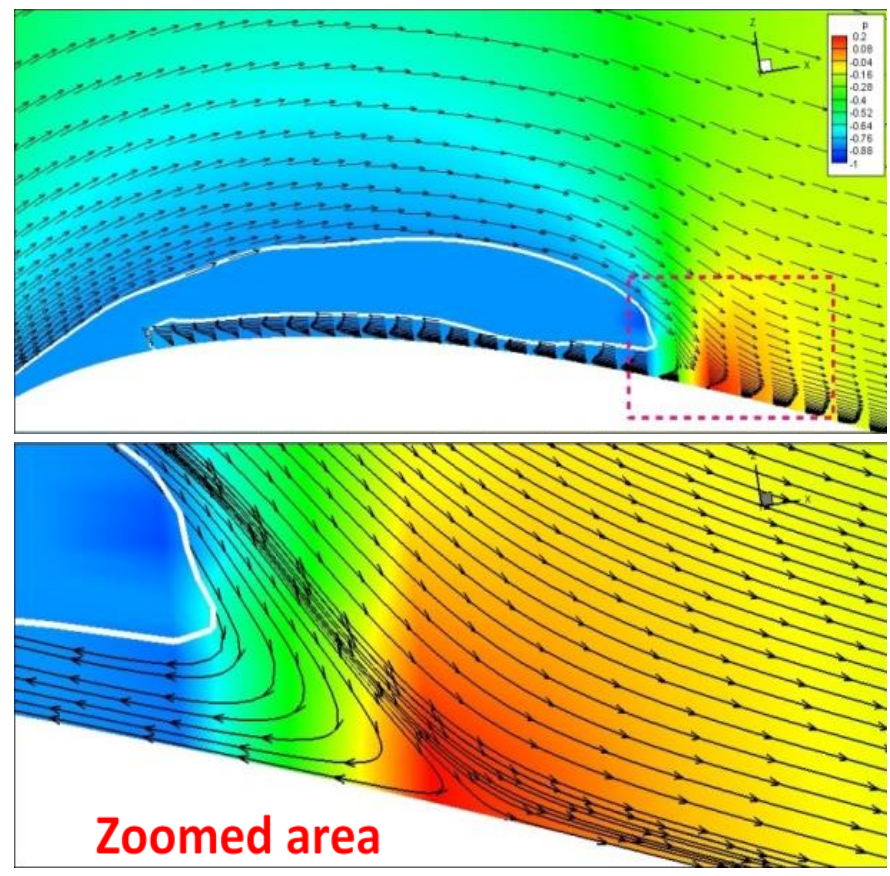

Figure 6: Zoom views of the velocity field and pressure contours around the sheet cavity (Top) and a zoom view of the red-boxed region of the streamlines in the reverse re-entrant jet flow region (Bottom).

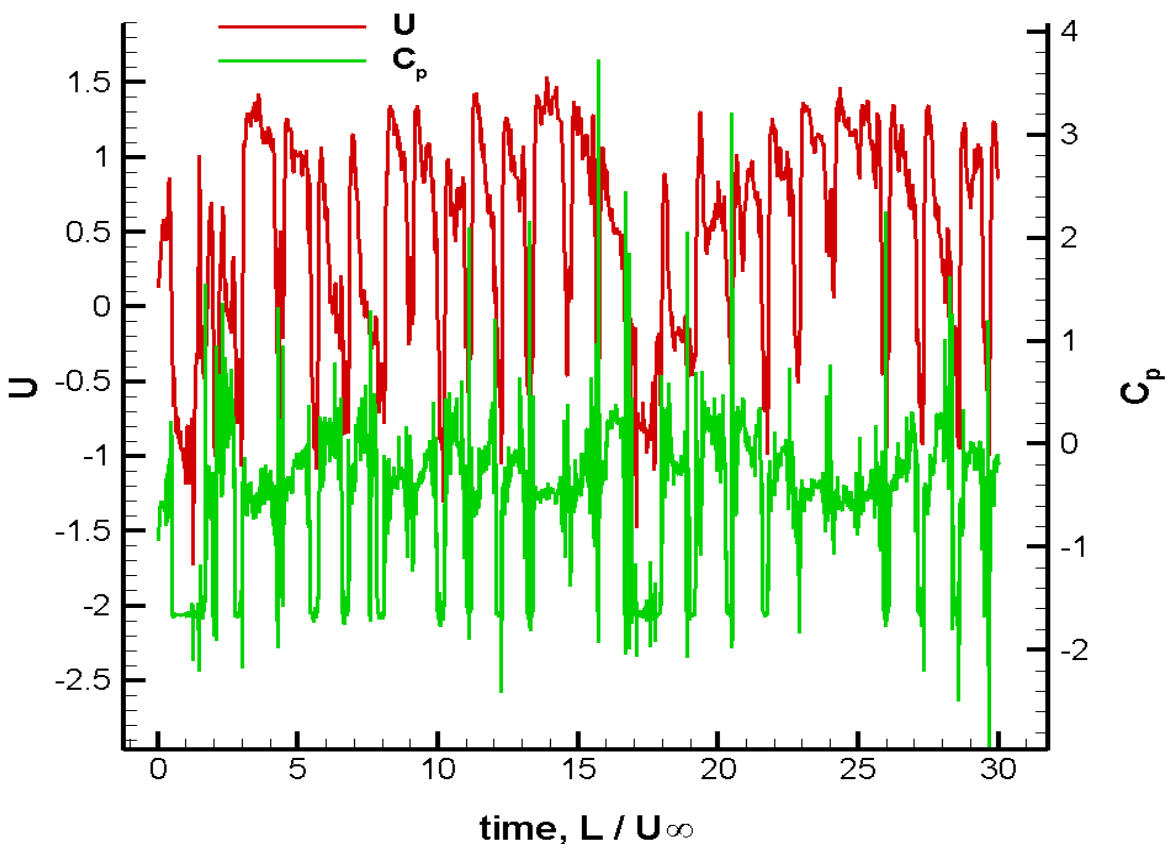

Figure 7: Time histories of the pressures and stream wise velocities at $x=0.4 L$ at the solid wall near the sheet cavity trailing edge. $\sigma=1.65$. 


\section{Sensitivity Study of Model Parameters}

\section{Nucleation Model Parameters}

The effects of the selection of the numerical values of the nucleation model parameters on the simulation results are examined here. The nucleation parameters $f_{n}$ and $N_{s}$, which express the number density and frequency of the nuclei emitted from the wall, as expressed by Equation (17), are investigated.

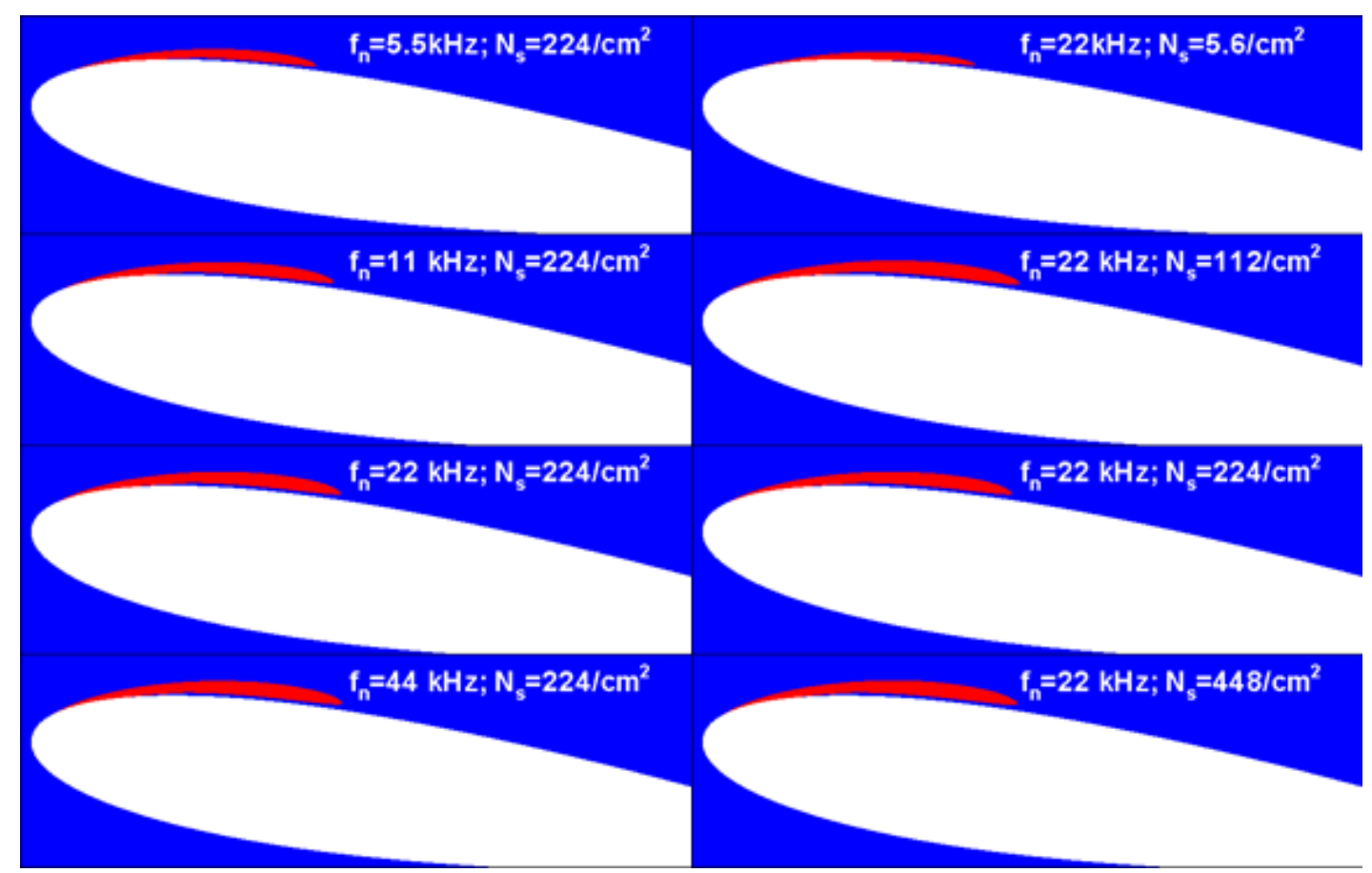

Figure 8: Effect of varying $f_{n}$ (left column) and $N_{s}$ (right column) on the time-averaged cavity shape for $\sigma=1.65$.

The left column of Figure 8 compares the time-averaged sheet cavity shapes for different assumed nucleation rates, $f_{\mathrm{n}}$, and for the same value of the number of nucleation sites per unit area, $N_{s}=224 \mathrm{~cm}^{-2}$. The right column of Figure 8, on the other hand, compares the averaged shapes for different assumed nucleation sites per unit area, $N_{\mathrm{s}}$, for the same value of the nucleation rate, $f_{n}=22 \mathrm{kHz}$. The same information is shown in quantitative form in Figure 9. It is seen that the time-averaged cavity length increases with the nucleation rate when $f_{\mathrm{n}}$ is small, but then remains almost unchanged for values of $f_{n}$ larger than $22 \mathrm{kHz}$. A similar trend can be seen when $N_{s}$ is increased for a constant nucleation frequency, $f_{\mathrm{n}}$. 

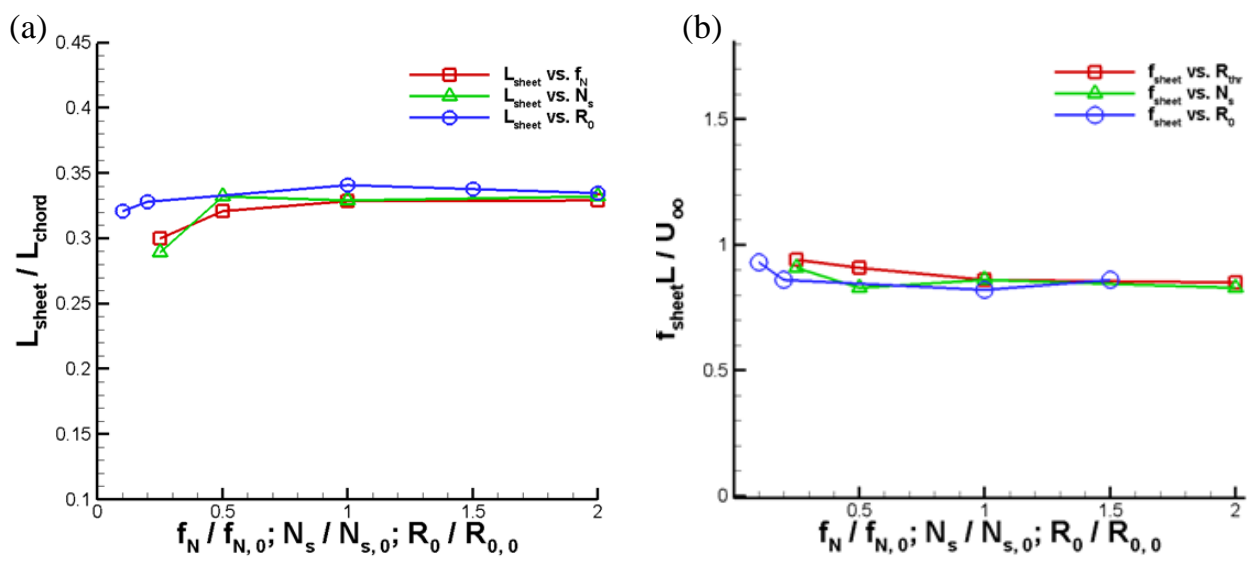

Figure 9: Effects of varying the wall nucleation parameters $f_{n}, N_{s}$ and $R_{0}$, on the normalized a) time-averaged cavity length, and b) cavity shedding frequency for $\sigma=1.65 .\left(f_{n, 0}=22 \mathrm{kHz}, N_{s, 0}=\right.$ $\left.224 / \mathrm{cm}^{2}, R_{0,0}=50 \mu \mathrm{m}\right)$

(a)

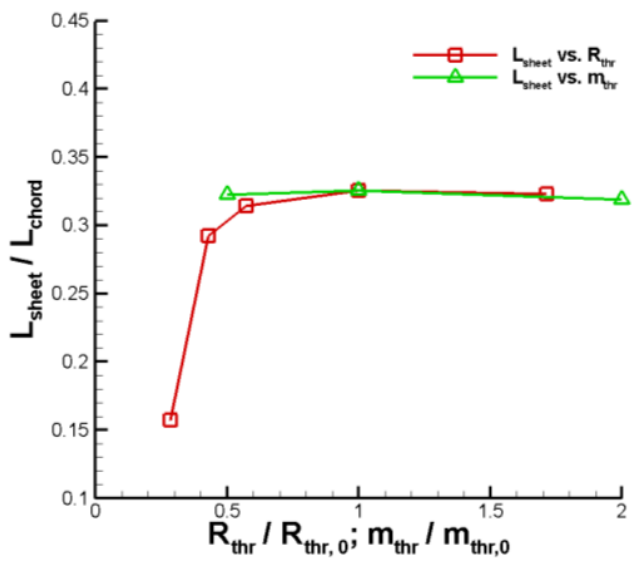

(b)

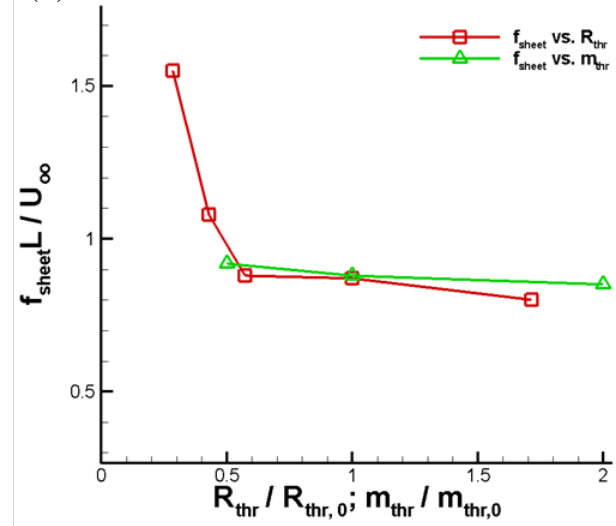

Figure 10: Effects of varying the threshold values, $R_{t h r}$ and $m_{t h r}$, in the switching from singular bubbles to large cavities, on the normalized a) time-averaged cavity length, and b) cavity shedding frequency $\sigma=1.65, R_{t h r, 0}=350 \mu \mathrm{m}$, and $m_{t h r, 0}=1$.

The time-averaged cavity length increases with the number density $N_{s}$ when $N_{s}$ is small, but then remains almost unchanged for values of $N_{s}$ larger than $112 \mathrm{~cm}^{-2}$. These trends imply that when the nucleation rate and nucleation sites per unit area are larger than some values, the cavity length reaches a saturation status and shows negligible dependency on those parameters. Since these two parameters are associated with surface roughness, the implication of the results is that surface treatment of the lifting surface can significantly influence the development of sheet cavitation. 
The same can be seen in Figure 9 concerning the effect of the selection of the radius, $R_{0}$, of the bubbles nucleated from the wall. For the whole range of radii tested between $10 \mu \mathrm{m}$ and $100 \mu \mathrm{m}$, both the cavity length and the frequency of oscillations remain approximately the same.

\section{Transition Model Parameters}

In the model for transition between micro scale and macro scale, criteria are introduced to switch discrete singularity bubbles to non-spherical deformable cavities represented by the zero level set. In order to demonstrate the generality of these criteria, a parametric study is conducted on the effects on the solution of the choice of the threshold values $R_{t h r}$ and $m_{t h r}$ in Equation (15). Figure 10 compares the time-averaged sheet length and the shedding frequency for different assumed threshold bubble radii, $R_{t h r}$, with the same value of the mesh factor, $m_{t h r}=1.0$. It is seen that the time-averaged cavity length increases as the threshold bubble radius increases when $R_{t h r}$ is small, but then remains almost unchanged when $R_{t h r}$ exceeds $200 \mu \mathrm{m}$. The same figure also shows little dependency on the threshold grid factor, $m_{t h r}$, for a constant $R_{t h r}=350 \mu \mathrm{m}$. The same conclusions apply for the predicted shedding frequency. This strongly indicates little dependency of the results on the switching criteria as long as the switching of the bubbles to a cavity is not done too early, i.e. when the bubbles have not grown significantly.

\section{Validation against Experimental Measurements}

In the following, the results of the present model are compared against published experimental data for different cavitation numbers and angles of attack. To do so, the cavitation number is varied in the computations by adjusting the far field pressure, $p_{\infty}$. Figure 11 compares the instantaneous pressures and stream wise velocities at $x=0.4 L$ for $\sigma=1.65$ and $\sigma=1.50$. Similar periodic oscillations of the local pressure and reversal of the jet velocity are seen in both cases. However, for $\sigma=1.50$ most of the time the stream wise velocity remains negative, but from time to time it is interrupted by sharp positive peaks. This is because, at this low cavitation number, the cavity extends further downstream than $x=0.4 \mathrm{~L}$. As a result, during most of the time this location is below the cavity and sees the reentrant jet. The sharp peaks appear when the sheet cavity breaks down due to the periodic shedding. This can be further explained by the pressure curves in Figure 11b. The pressure mostly stays at the vapor pressure, $C_{p}=-1.5$, with repeated peak pressure increases at a nearly constant frequency. The observation point for the $\sigma=1.65$ case does not see vapor pressure but still observes the repeated pressure peaks at a frequency slightly higher than that for $\sigma=1.50$. This is made apparent in Figure 12, which shows the frequency spectrum of the signal 
through a Fast Fourier Transform (FFT) analysis of both the re-entrant jet velocity and the pressure oscillations.
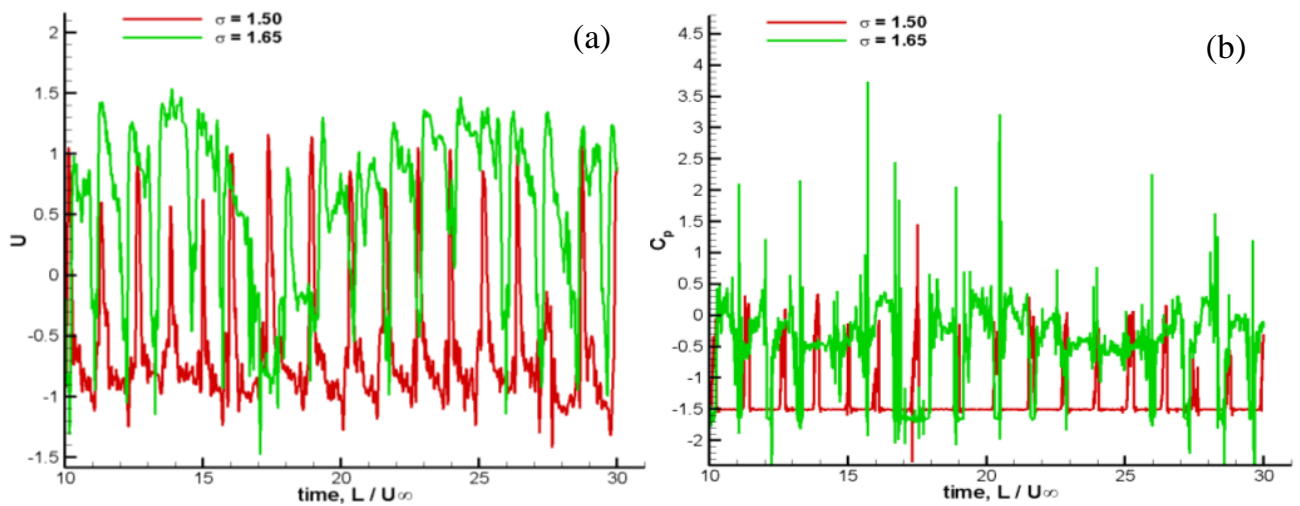

Figure 11: Comparison for $\sigma=1.65$ and $\sigma=1.50$ of the time histories of a) the stream wise velocity and $\mathrm{b}$ ) the instantaneous pressure monitored at $x=0.4 L$ on the NACA 0015 foil. $\alpha=8^{\circ}$, $\mathrm{U}_{\infty}=10 \mathrm{~m} / \mathrm{s}, L=0.12 \mathrm{~m}$.

Quantitative comparisons of these frequencies with the experimental data, which measured cloud shedding frequencies for three different cavitation numbers, $\sigma=1.50,1.65$ and 1.80 and two different angle of attacks, $\alpha=8^{\circ}$ and $10^{\circ}$, are displayed in Figure 13. The results are presented in terms of the non-dimensional shedding frequency, $f_{\text {sheet }} L / U_{\infty}$, versus the ratio of the cavitation number and the angle of attack, $\sigma / 2 \alpha$. A very good agreement can be seen between the numerical predictions and the experimental measurements in the simulated range.

In addition to the shedding frequency, the time-averaged cavity lengths deduced from the unsteady simulations are also compared to the experimental measurements. Figure 14 illustrate the time-averaged cavity shape deduced for $\sigma=1.65$ and $\sigma=1.50$ by averaging the solution from the unsteady simulations after excluding the initial transient period. Figure 15 shows a comparison with the results summarized in Berntsen et al. 2001. The figure shows a very good agreement between numerical predictions and experimental measurements for the time-averaged cavity length for three different cavitation numbers, $\sigma=1.50,1.65$ and 1.80 and two angle of attacks, $\alpha=8^{\circ}$ and $10^{\circ}$. 

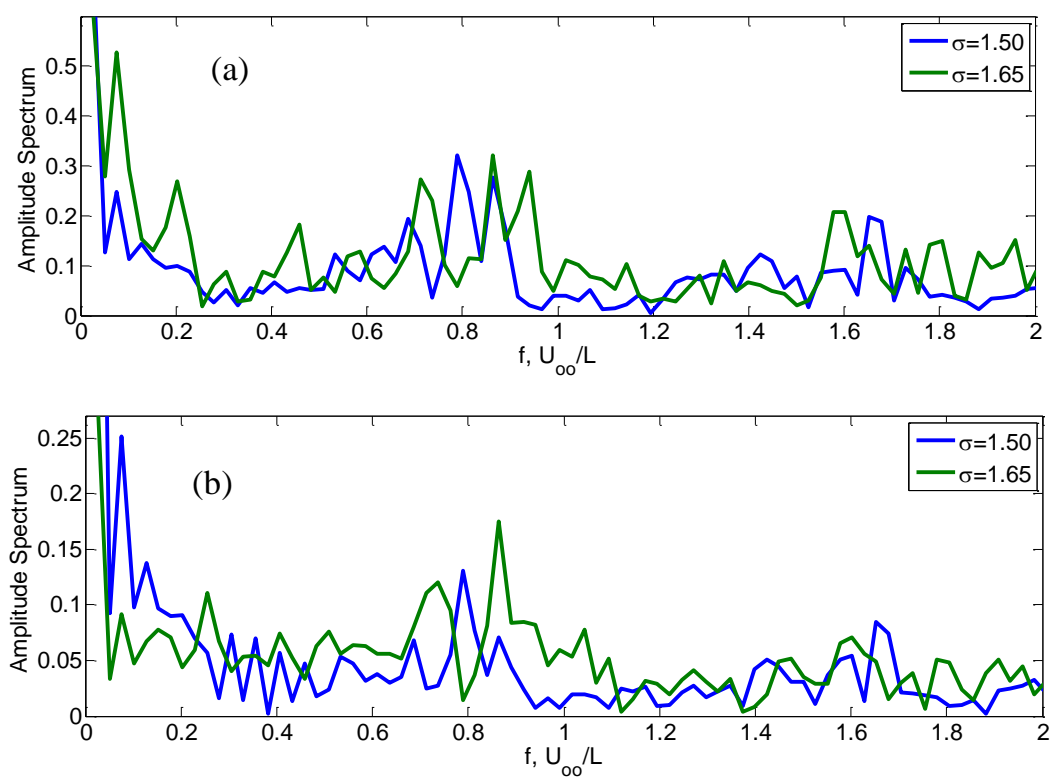

Figure 12: Comparison between the cases of $\sigma=1.65$ and $\sigma=1.50$ of the frequency spectra of the temporal variations of the stream wise velocity (a) and of the pressure (b) monitored at $x=0.4 \mathrm{~L}$ at the solid wall on the suction side.

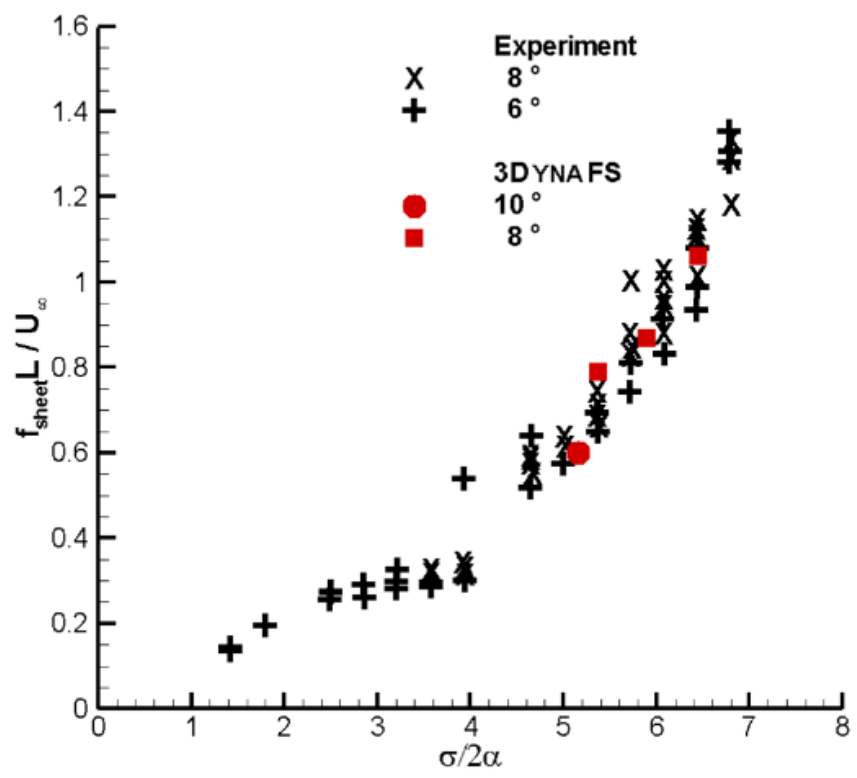

Figure 13: Comparison of the shedding frequencies of the sheet cavity on the NACA0015 for $\alpha=8^{\circ}, U_{\infty}=10 \mathrm{~m} / \mathrm{s}, L=0.12 \mathrm{~m}$ with the experimental results summarized in Berntsen et al. 2001 . 


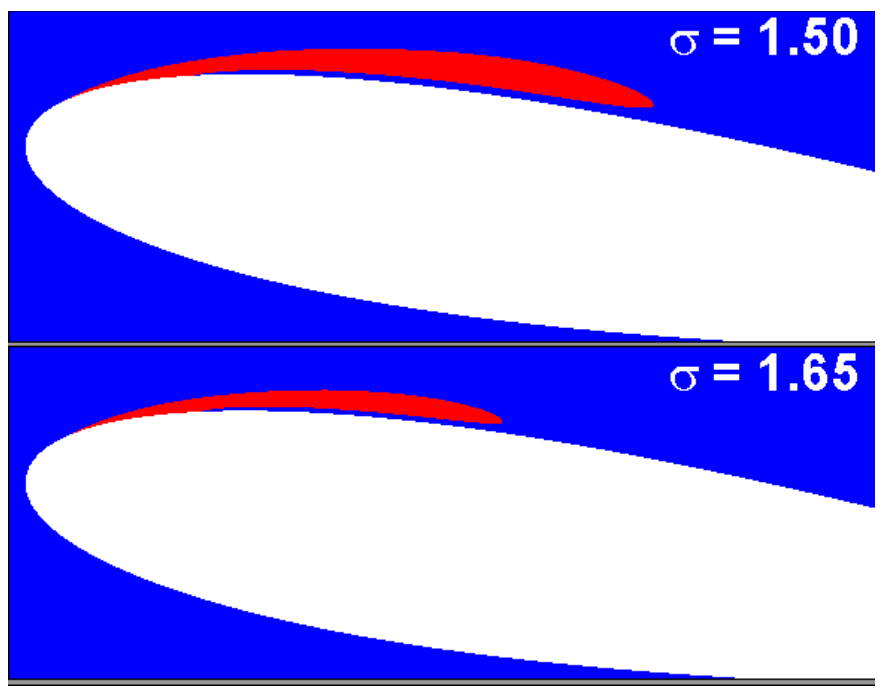

Figure 14: Comparison of the time-averaged cavity shapes between $\sigma=1.65$ and $\sigma=1.50$ for the NACA 0015 hydrofoil. $\alpha=8^{\circ}, \mathrm{U}_{\infty}=10 \mathrm{~m} / \mathrm{s}, L=0.12 \mathrm{~m}$.

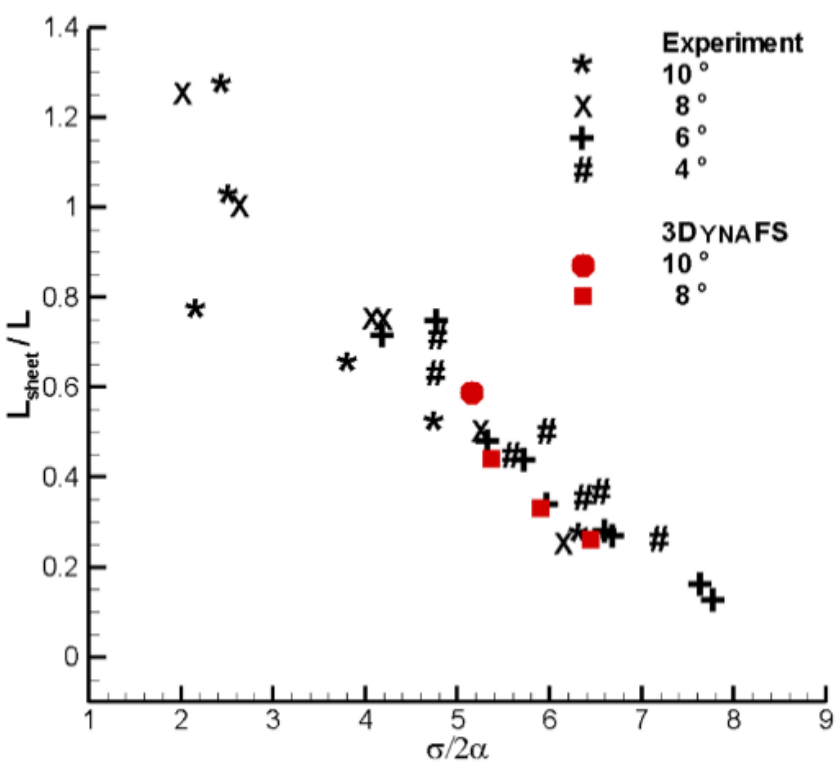

Figure 15: Comparison of the time-averaged cavity length on the NACA0015 for $\alpha=8^{\circ}, U_{\infty}=10$ $\mathrm{m} / \mathrm{s}, L=0.12 \mathrm{~m}$ with the experimental results summarized in Berntsen et al. 2001 .

\section{APPLICATION TO 3D LIFTING SURFACES}

To test capability of the developed multiscale two-phase flow model for simulations of sheet and cloud cavitation on 3D lifting surface we present 3D simulation cases with different levels of complexity. 


\section{D Computation of the NACA0015 Foil}

A 3D computation on a hydrofoil having the same foil shape as the 2D NACA0015 shown above, but with a span equal to one quarter of the chord length is considered. The foil uses the same computational domain and grid resolution as for the $2 \mathrm{D}$ computation except that 20 grid points are now used in the span wise direction which is bounded by two side walls. The same boundary conditions as in previous sections are applied for the far field boundaries and a slip boundary condition is imposed on both side walls in the $3 \mathrm{D}$ simulation.

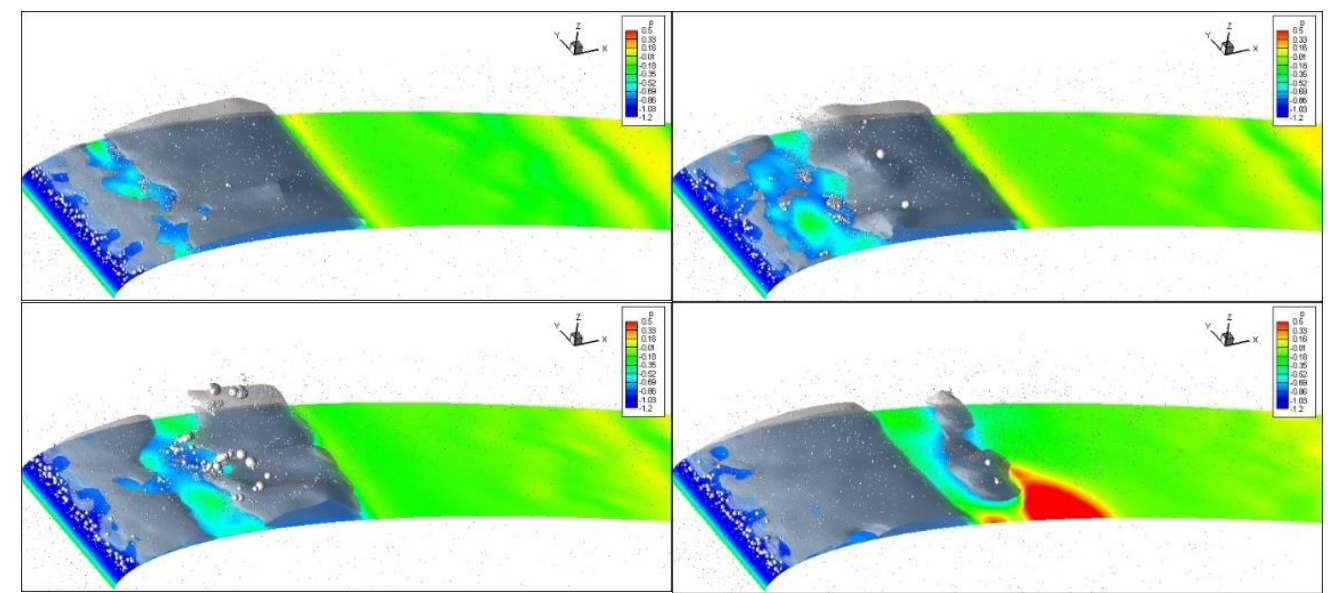

Figure 16: Time sequence of the 3D sheet cavity dynamics on a NACA0015 hydrofoil for $\sigma=1.65$, $\alpha=8^{\circ}, U_{\infty}=10 \mathrm{~m} / \mathrm{s}, L=0.12 \mathrm{~m}$.

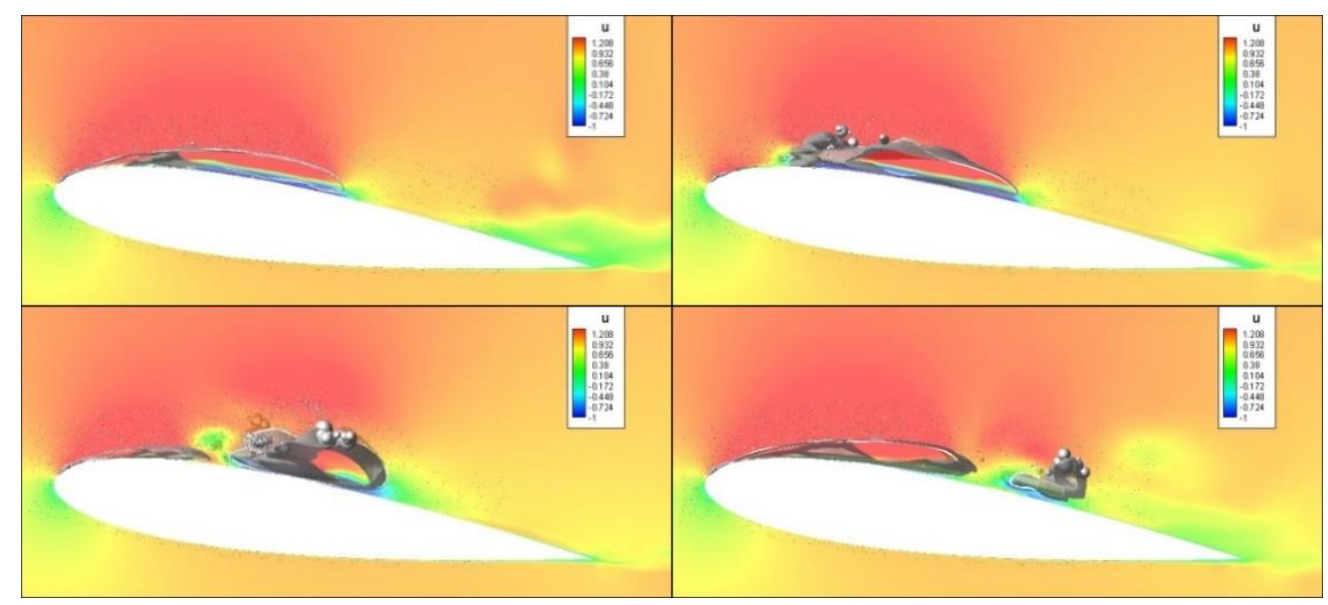

Figure 17: 2D projected side views of a time sequence of 3D simulations of an oscillating sheet cavity on a NAC0015 hydrofoil for $\sigma=1.65, \alpha=8^{\circ}, U_{\infty}=10 \mathrm{~m} / \mathrm{s}, L=0.12 \mathrm{~m}$. 
A time sequence of sheet cavity development, oscillations, and bubble cloud shedding on the foil for $\sigma=1.65$ and $\alpha=8^{\circ}$ is shown in Figure 16 and Figure 17. It is seen that although the cloud shedding dynamics is similar to that observed in the $2 \mathrm{D}$ computations, i.e. the sheet cavity starts near the leading edge and gradually evolves until a reentrant jet breaks the cavity into parts and forms a detached bubble cloud, the 3D computations exhibit a more complicated breakup, i.e. the cavity breakup location varies in the span wise direction due to the 3D nature of the unsteady flow.

\section{D Oscillating Finite-Span Hydrofoil}

\section{Case Setup}

In this section we consider an oscillating finite-span hydrofoil for which experimental data is available from Boulon (1996). This concerns an elliptic hydrofoil having a NACA16020 crosssection and a root chord length of $L=6 \mathrm{~cm}$ with an aspect ratio of 1.5 (based on semi-span). In order to simulate the hydrofoil oscillating in an overall fixed fluid frame, a moving overset grid scheme is applied to the neighborhood of the foil. As illustrated in Figure 18 a 12-block grid is generated to encompass the finite-span foil in a domain bounded by boundaries located at about one chord length away from the foil surface. This 12-block grid is then overset over a fixed background grid which has domain boundaries located at about 5 times of chord length away from the foil surface as shown in Figure 19. A total of 0.25 million grid points was generated for the overset grids with a first grid spacing of $1 \times 10^{-4}$ chord length in the normal direction to the foil surface.

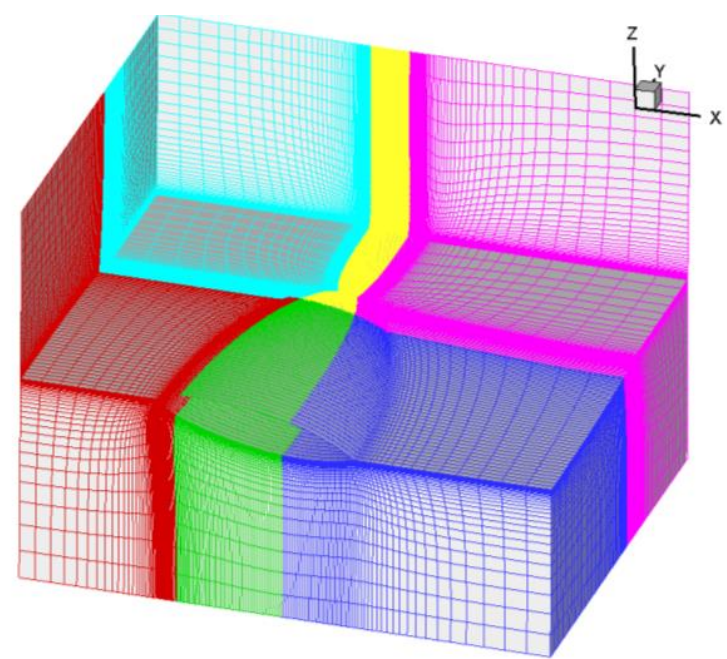

Figure 18: Overset 12-block grid generated for a finite-span NACA16020 elliptic hydrofoil. 


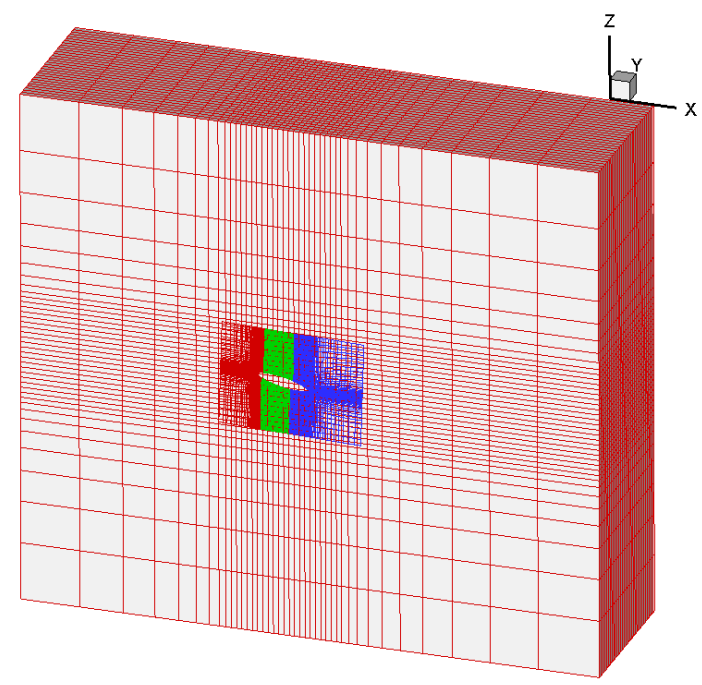

Figure 19: Overset grids with a 12-block moving grid over a fixed background grid used for the simulation of sheet cavitation and cloud shedding on an oscillating hydrofoil.

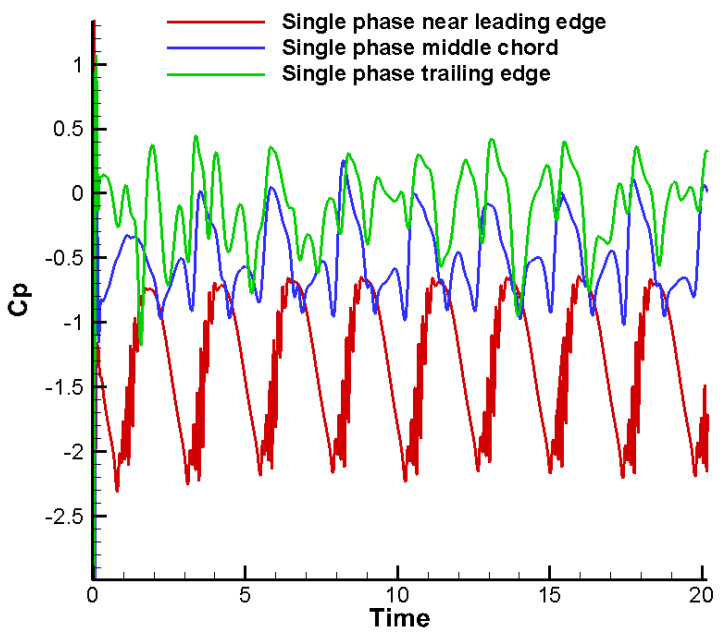

Figure 20: Time histories of the pressure coefficient monitored at three locations, $x=0.04 L$ (near the leading edge) and $x=0.5 L$ (at middle chord) and $x=L$ (at the trailing edge).

\section{Single-Phase Flow Computations}

We present the case of the hydrofoil in a uniform flow field at a velocity of $3.33 \mathrm{~m} / \mathrm{s}$ oscillating with a time-dependent angle of attack, $\alpha(t)$, prescribed by the sinusoidal function:

$$
\alpha(t)=\alpha_{0}+\Delta \alpha \sin (2 \pi f t)
$$


where the initial angle of attack $\alpha_{0}$ is $10^{\circ}$. The oscillations amplitude is $\Delta \alpha=5^{\circ}$ and the oscillations frequency is $f=23 \mathrm{~Hz}$. The single phase flow field was first simulated until the solution reached limit cycle oscillations. Figure 20 shows the time histories of the pressure coefficient monitored at three locations, $x=0.04 L$ (near leading edge) and $x=0.5 L$ (middle chord) and $\quad x=L$ (at the trailing edge). It is seen that the pressure has reached a repeated limit cycle oscillation at the leading edge and the mid chord locations. However, the pressure at the trailing edge location still shows irregular vortex pairing occurring upstream.

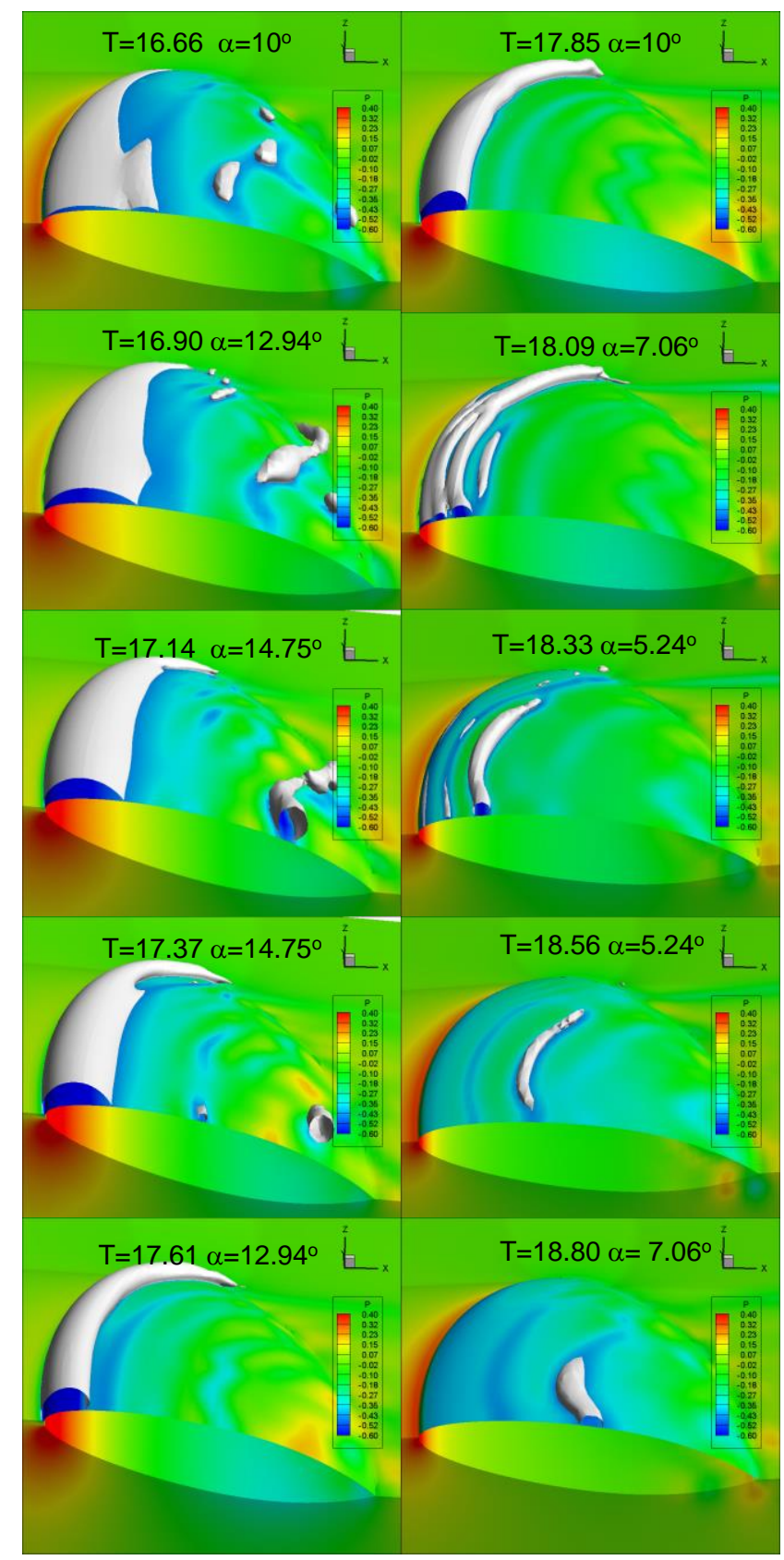


Figure 21: A time sequence of the pressure contours and an iso-pressure surface $\left(p=p_{v}\right)$ during one oscillating cycle of the finite span NACA16020 elliptic hydrofoil after the unsteady solution reached limit cycle oscillation.

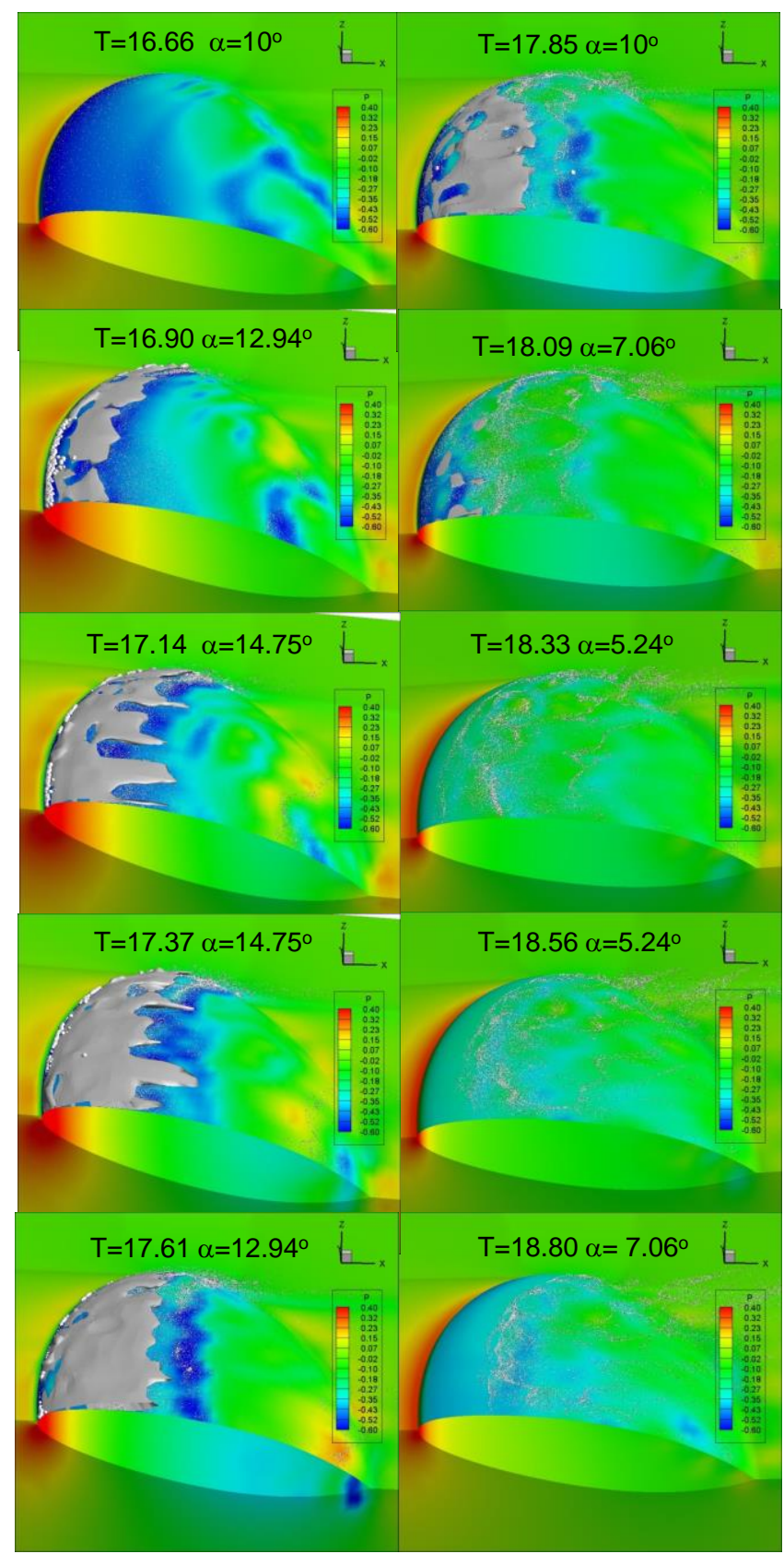

Figure 22: A time sequence of sheet cavity evolving on the suction side of an oscillating finitespan hydrofoil obtained for $\sigma=0.9$.

Figure 21 shows pressure contours and the iso-pressure surface $p=p_{v}$ for one oscillating cycle. It is seen that a low pressure region where the flow separation occurs starts to form near the 
leading edge as the angle of attack increases during the cycle. The low pressure region extends downstream and reaches a maximum length before the hydrofoil reaches the highest angle of attack. The length of the low pressure region reduces but its height increases as the hydrofoil reaches the maximum angle of attack. The low pressure region starts to shrink as the angle of attack is decreased from the highest value and the low pressure region reduced to the smallest size when the angle of attack reaches the lowest value. Strong well defined vortical regions are seen to propagate downstream form the leading edge.

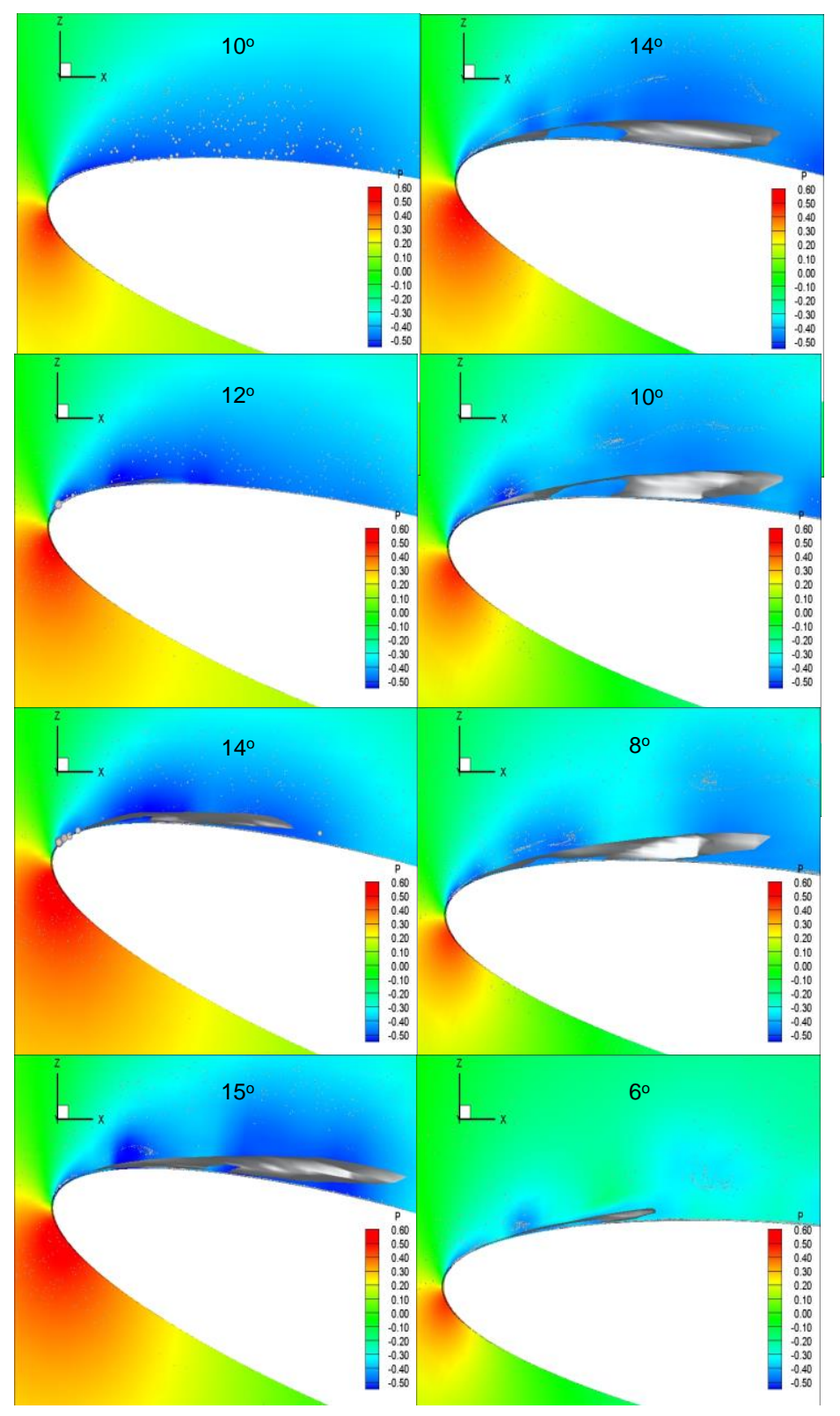

Figure 23: Zoomed view of the root sheet cavity near the leading edge obtained for $\sigma=0.9$. 

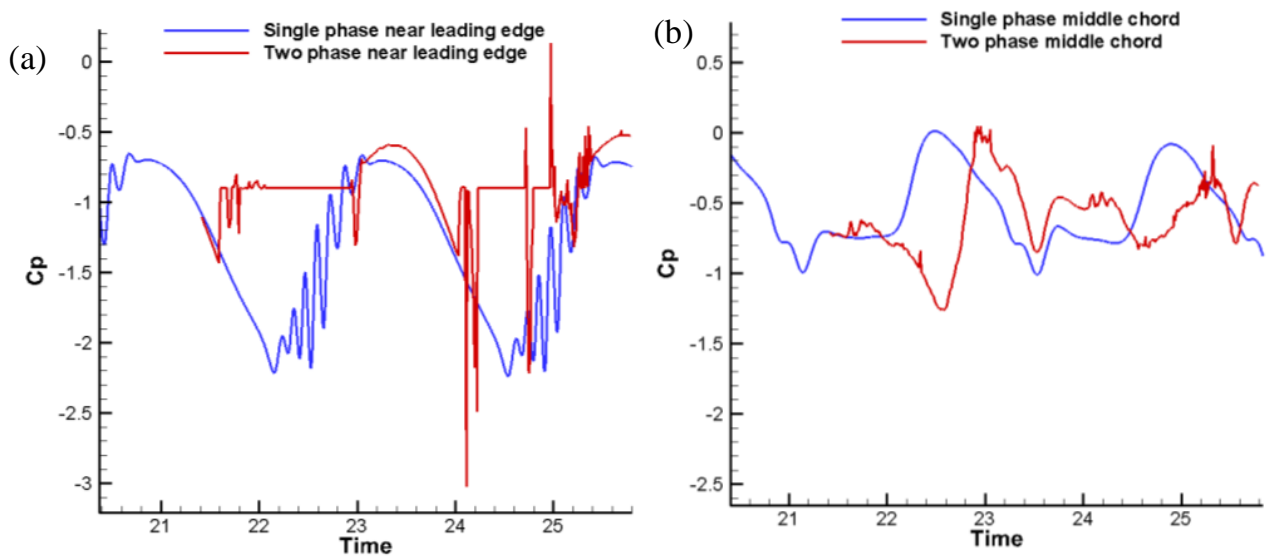

Figure 24: Comparison of time histories of pressure coefficients monitored at (a) $x=0.04 L$ (near the leading edge) and (b) $x=0.5 \mathrm{~L}$ (mid-chord) for single phase and two-phase flow computations.

\section{Simulation of Sheet and Cloud Cavitation}

Two-phase simulation to capture sheet and cloud cavitation was initiated once the single phase flow solution reached limit cycle oscillation. Free nuclei were allowed to propagate in the liquid, while nucleation was also activated at the foil boundary as in the $2 \mathrm{D}$ study shown earlier. Figure 22 shows a time sequence of the sheet cavitation evolving on the suction side of the finite-span hydrofoil for $\sigma=0.9$.

A zoomed view of the root sheet cavity near the leading edge is also shown in Figure 23 for a better illustration. It is seen that the sheet cavity starts to form from the leading near the tip region. It then extends toward the root as the angle of attack increases. However, unlike the low pressure region observed in the single phase flow solution, the sheet cavity does not reach its maximum extent before the hydrofoil reaches the highest angle of attack. On the contrary, the sheet cavity continues to grow beyond the time the foil has the highest angle of attack. After reaching its maximum extent, the sheet cavity subsides without showing clear cloud shedding in this case. This could be due to an inadequate grid resolution used in this simulation. Simulations with a much finer grid may be required for a better prediction of the sheet cavity.

Figure 24 shows a comparison of the time histories of the pressure coefficient monitored at $x=0.04 \mathrm{~L}$ (near leading edge) and $x=0.5 \mathrm{~L}$ (middle chord) between the single-phase and the twophase flow computations. The modifications of the pressure due to the sheet cavity can be clearly seen at both locations. For the location near the leading edge, the pressure at the hydrofoil surface becomes equal to the vapor pressure $\left(C_{p}=-0.9\right)$ once the sheet cavity reaches this location. Pressure 
spikes are also observed as in the 2D computations due to the local breakup of the sheet cavity. For the location at the middle chord, the pressure is also changed by the presence of the unsteady cavity. However, the pressure does not reach vapor pressure because the sheet cavity length does not reach this location.

\section{CONCLUSIONS}

A multiscale framework was developed for the simulation of unsteady sheet cavitation on a hydrofoil by smoothly bridging a Level Set method (LSM) for large size cavities and a Discrete Singularity Model (DSM) for small bubbles. Starting with the micro-scale physics of nucleation from the solid surface and dispersed nuclei in the bulk liquid, sheet cavitation along a NACA0015 hydrofoil is well captured by the model. The predicted sheet lengths and oscillation frequencies under several cavitation numbers match the experimental data very well. Parametric studies indicate that when the nucleation rate and nucleation sites per area are not too small, the cavity characteristics show negligible dependency on those parameters. Sensitivity assessment of the threshold values of the switch criteria between discreet microbubbles and large cavities also shows little dependency on the parameters as long as the size criterion for transforming a nuclei bubble into a large cavity is not too small.

The developed multiscale two-phase flow model was also applied to simulate sheet and cloud cavitation on 3D lifting surface. Three-dimensional simulations for the 2D NACA0015 foil capture well sheet cavitation dynamics and cloud shedding with a more complicated breakup observed due to the $3 \mathrm{D}$ unsteadiness of the flow.

Sheet cavitation on an oscillating finite-span hydrofoil was also simulated using the developed model and a moving overset grid scheme. Although the unsteady development of the sheet cavity was also captured, the result do not show yet clear cloud shedding probably due to inadequate grid resolution. This will be improved by refining the grids and conducting longer duration simulations.

\section{ACKNOWLEDGMENTS}

This work was supported by the Office of Naval Research under contract N00014-12-C-0382 monitored by Dr. Ki-Han Kim. This support is highly appreciated. 


\section{REFERENCES}

Atchley, A.A. and A. Prosperetti, "The Crevice Model of Bubble Nucleation," The Journal of the Acoustical Society of America, 86(3): p. 1065-1084, 1989.

Balachandar, S. and J.K. Eaton, "Turbulent Dispersed Multiphase Flow,” Annual Review of Fluid Mechanics, 42: p. 111-133, 2010.

Berntsen, G.S., M. Kjeldsen, and R.E. Arndt. "Numerical Modeling of Sheet and Tip Vortex Cavitation with FLUENT 5," in Fourth International Symposium on Cavitation. Pasadena, CA: NTNU, 2001.

Biesheuvel, A. and van Wijngaarden, L. "Two-Phase Flow Equations for a Dilute Dispersion of Gas Bubble in Liquid”, Journal of Fluid Mechanics, Vol. 148, pp. 301-318, 1984.

Billet, M. L., "Cavitation Nuclei Measurements - A Review," ASME Cavitation and Multiphase Flow Forum," FED Vol. 23, June 1985.

Briggs, L.J., "Limiting Negative Pressure Of Water," Journal of Applied Physics, 21(7): p. 721722, 2004.

Boulon, O., "Etude Expérimentale de la Cavitation de Tourbillon Marginal - Effets Instationnaires de Germes et de Confinement, ” Thèse, Institut National Polytechnique de Grenoble, 1996.

Browne, C., et al., "Bubble Coalescence During Acoustic Cavitation in Aqueous Electrolyte Solutions," Langmuir, 27(19): p. 12025-12032, 2011.

Chahine, G.L. and R. Duraiswami, "Dynamical Interactions in a Multi-Bubble Cloud," Journal of fluids engineering, 1992. 114(4): p. 680-686.

Chahine, G.L., "Strong Interactions Bubble/Bubble and Bubble/Flow," in Bubble Dynamics and Interface Phenomena, Springer. p. 195-206, 1994.

Chahine, G.L. and K. Kalumuck, "BEM Software for Free Surface Flow Simulation Including Fluid-Structure Interaction Effects,” International Journal of Computer Applications in Technology, 11(3): p. 177-198, 1998.

Chahine, G.L. and Hsiao, C-T. "Modeling 3-D Unsteady Sheet Cavities Using a Coupled UnRANS-BEM Code." Proceedings of the 23rd Symposium on Naval Hydrodynamics, Val De Reuil, France, September 17-22, 2000.

Chahine, G.L., "Nuclei Effects on Cavitation Inception and Noise”, Keynote presentation, 25th Symposium on Naval Hydrodynamics, St. John's, Newfoundland, Canada, Aug. 8-13, 2004. 
Chahine, G.L., "Numerical Simulation of Bubble Flow Interactions," Journal of Hydrodynamics, Ser. B, 21(3): p. 316-332, 2009.

Chen, Y. and Heister, D.D., “A Numerical Treatment for Attached Cavitation,” Journal of Fluids Engineering, 116, pp 613-618, 1994.

Choi, J.-K., Hsiao, C.-T., and Chahine, G.L., "Tip Vortex Cavitation Inception Study Using the Surface Averaged Pressure (SAP) Model Combined with a Bubble Splitting Model”, 25th Symposium on Naval Hydrodynamics, St. John's, NL, Canada, 2004.

Dabiri, S., Sirignano, W.A. and Joseph, D.D., "Cavitation in an Orifice Flow," Physics of Fluids, 19, 2007.

Dabiri, S., Sirignano, W.A. and Joseph, D.D. "Two-Dimensional and axisymmetric Viscous Flow in Apertures," J. Fluid Mech., 605, pp. 1-18, 2008.

Deshpande, M., Feng, J., and Merkle, C.L., "Navier-Stokes Analysis of 2D Cavity Flows," Cavitation and Multiphase Flow FED-Vol. 153, pp 149-155, 1993.

Druzhinin, O.A. and Elghobashi, S., "Direct Numerical Simulations of Bubble-Laden Turbulent Flows Using the Two-Fluid Formulation, ” Physics of Fluids, Vol. 10, pp. 685-607, 1998.

Druzhinin, O.A. and Elghobashi, S., “A Lagrangian-Eulerian Mapping Solver for Direct Numerical Simulation of Bubble-Laden Turbulent Shear Flows Using the Two-Fluid Formulation,” Journal of Computational Physics, Vol. 154, p. 174-196, 1999.

Druzhinin, O.A. and Elghobashi, S., "Direct Numerical Simulation of a Three-Dimensional Spatially Developing Bubble-Laden Mixing Layer with Two-Way Coupling," Journal of Fluid Mechanics, Vol. 429, p. 23-62, 2001.

Fedkiw, R.P., Aslam, T., Merriman, B. and Osher, S., "A Non-Oscillatory Eulerian Approach to Interfaces in Multimaterial Flows (The Ghost Fluid Method)," Journal of Computational Physics, Vol.169, pp. 463-502, (1999).

Franklin, R.E., "A Note on the Radius Distribution Function for Microbubbles of Gas in Water," ASME Cavitation and Multiphase Flow Forum, FED-Vol. 135, pp.77-85, 1992.

Haberman, W.L., and Morton, R.K., “An Experimental Investigation of the Drag and Shape of Air Bubble Rising in Various Liquids,” DTMB Report 802, 1953.

Harvey, E.N., et al., "Bubble Formation in Animals. II. Gas Nuclei and Their Distribution in Blood and Tissues," Journal of Cellular and Comparative Physiology, 24(1): p. 23-34, 1944. 
Hirschi, R., Dupont, P., and Avellan, F., "Partial Sheet Cavities Prediction on a Twisted Elliptical Platform Hydrofoil Using a Fully 3-D Approach, ” Proceedings 3rd international Symposium on Cavitation, Grenoble, France, Vol. 1, pp 2450249, 1998.

Hsiao, C.-T. and G. Chahine, "Prediction of Tip Vortex Cavitation Inception Using Coupled Spherical and Nonspherical Bubble Models and Navier-Stokes Computations," Journal of marine science and technology, 8(3): p. 99-108, 2004.

Hsiao, C.-T. and G. Chahine, "Numerical Study of Cavitation Inception Due to Vortex/Vortex Interaction in a Ducted Propulsor,” Journal of Ship Research, 52(2): p. 114-123, 2008.

Hsiao, C.-T., Chahine, G.L., and Liu, H.-L., "Scaling Effects on Prediction of Cavitation Inception in a Line Vortex Flow,” Journal of Fluid Engineering, Vol. 125, 2003, pp.53-60.

Hsiao, C.-T., Chahine, G.L., and Liu, H.-L. "Scaling Effect on Prediction of Cavitation Inception in a Line Vortex Flow,” Journal of fluids engineering, 125(1): p. 53-60, 2003.

Hsiao, C.T., Wu, X., Ma, J., and Chahine, G.L., "Numerical and Experimental Study of Bubble Entrainment Due to a Horizontal Plunging Jet," International Shipbuilding Progress, 60(1): p. 435469, 2013.

Kang, M., Fedkiw and Liu, L.D., "A Boundary Condition Capturing Method for Multiphase Incompressible Flow“, J. Sci. Comp., Vol. 15, pp. 323-360, 2000,

Kawamura T., and Sakoda M., "Comparison of Bubble and Sheet Cavitation Models for Simulation of Cavitating Flow over a Hydrofoil", Fifth International Symposium on Cavitation, November 1-4, Osaka, Japan, 2003.

Kinnas, S.A. and Fine, N.E. "A Numerical Nonlinear Analysis of the Flow around Two- and ThreeDimensional Partially Cavitating Hydrofoils,” J Fluid Mech, 254. 1993.

Kinzel, M.P., Lindau, J.W., Kunz, R.F., “A Level-Set Approach for Large Scale Cavitation,” DoD High Performance Computing Modernization Program Users Group Conference, San Diego, CA, June 15-18, 2009.

Y. Kodama, N. Take, S. Tamiya and H. Kato "The Effect of Nuclei on the Inception of Bubble and Sheet Cavitation on Axisymmetric Bodies,” J. Fluids Eng. 103(4), 557-563, Dec 01, 1981.

Kubota, A., Hiroharu, K., Yamaguchi, H., "A New Modeling of Cavitating Flows: A Numerical Study of Unsteady Cavitation on a Hydrofoil Section”, Journal of Fluid Mechanics, Vol. 240, pp. 59-96, 1992. 
Kuiper, G. “Cavitation in Ship Propulsion,” TU Delft, Jan. 15, 2010.

Kunz, R.F. Boger, D.A., Stinebring, D.R., Chyczewski, T.S., Lindau, J.W. and Gibeling, H.J. “A Preconditioned Navier-Stokes Method for Two-Phase Flows with Application to Cavitation" Computers \& Fluids, Vol. 29, pp. 849-875, 2000.

Ma, J., et al., "Two-Fluid Modeling of Bubbly Flows Around Surface Ships Using a Phenomenological Subgrid Air Entrainment Model.” Computers \& Fluids, 52: p. 50-57, 2011.

Ma, J., et al., "A Two-Way Coupled Polydispersed Two-Fluid Model for the Simulation of Air Entrainment beneath a Plunging Liquid Jet,” ASME Journal of Fluids Engineering, 134(10), 2012.

Ma, J., Hsiao, C.-T. and Chahine, G.L., "Euler-Lagrange Simulations of Bubble Cloud Dynamics near a Wall”, ASME Journal of Fluid Engineering, 137(4), pp.041301.

Medwin, H., “Counting Bubbles Acoustically: A Review,” Ultrasonics, 15, 7-13, 1977.

Merkle, C.L., Feng, J., and Buelow, P.E.O., "Computational Modeling of the Dynamics of Sheet Cavitation," in Proceedings of the 3rd International Symposium on Cavitation, (CAV1998), Grenoble, France, 1998.

Mørch, K.A., "Cavitation Nuclei: Experiments and Theory," Journal of Hydrodynamics, Ser. B, 21(2): p. 176-189, 2009.

Osher, S. and Fedkiw, R.P., "Level Set Methods, an Overview and Some Recent Results," Journal of Computational Physics, Vol.169, pp. 463-502, 2001.

Prince, M.J. and H.W. Blanch, "Bubble Coalescence and Break-Up in Air-Sparged Bubble Columns," AIChE Journal, 36(10): p. 1485-1499, 1990.

Raju, R., Singh, S., Hsiao, C.-S., and Chahine, G., "Study of Pressure Wave Propagation in a TwoPhase Bubbly Mixture," Transactions of the ASME-I-Journal of Fluids Engineering, 133(12): p. 121302, 2011.

Roe, P.L., “Approximate Riemann Solvers, Parameter Vectors, and Difference Schemes,” Journal of Computational Physics, Vol. 43, pp. 357-372, 1981.

Saffman, P.G., "The Lift on a Small Sphere in a Slow Shear Flow", Journal of Fluid Mechanics, Vol. 22, pp.385-400. 1965

Singhal, N.H., Athavale, A.K., Li, M. and Jiang, Y. "Mathematical Basis and Validation of the Full Cavitation Model” J. of Fluids Engineering, Vol. 124, pp. 1-8, 2002. 
Shen, Y. and P. Dimotakis, "The Influence of Surface Cavitation on Hydrodynamic Forces," in American Towing Tank Conference, 22nd. 1989.

van Leer, B., and Woodward, P.R., "The MUSCL Code for Compressible Flow: Philosophy and Results”, B van Leer, PR Woodward - Proc. of the TICOM Conf., Austin, TX, 1979.

Vanden, K.J., and Whitfield, D.L., "Direct and Iterative Algorithms for the Three-Dimensional Euler Equations", AIAA Journal, Vol. 33, No. 5, pp. 851-858, 1995.

Varghese A.N., Uhlman, J.S., Kirschner, I.N., "High Speed Bodies in Partially Cavitating Axisymmetric Flow,” Journal of Fluids Eng., 127, pp 41-54, 2005.

Shams, E., J. Finn, and S. Apte, "A Numerical Scheme for Euler-Lagrange Simulation of Bubbly Flows in Complex Systems," International Journal for Numerical Methods in Fluids, 67(12): p. 1865-1898, 2011.

Ferrante, A. and S. Elghobashi, "On the Physical Mechanisms of Drag Reduction in a Spatially Developing Turbulent Boundary Layer Laden with Microbubbles,” Journal of Fluid Mechanics, 503: p. 345-355, 2004.

Xu, J., M.R. Maxey, and G.E. Karniadakis, "Numerical Simulation of Turbulent Drag Reduction Using Micro-Bubbles,” Journal of Fluid Mechanics, 468: p. 271-281, 2002.

Yount, D.E., "Skins of Varying Permeability: A Stabilization Mechanism for Gas Cavitation Nuclei," The Journal of the Acoustical Society of America, 65(6): p. 1429-1439, 1979.

Yount, D., E. Gillary, and D. Hoffman, "A Microscopic Investigation of Bubble Formation Nuclei," The Journal of the Acoustical Society of America, 76(5): p. 1511-1521, 1984.

Yuan, W., Sauer J., and Schnerr, G.H., "Modeling and Computation of Unsteady Cavitation Flows in Injection Nozzles,” J. of Mechanical Ind., Vol. 2, pp. 383-394, 2001.

Wu, X., and Chahine, G.L. "Development of an Acoustic Instrument for Bubble Size Distribution Measurement", Journal of Hydrodynamics, Ser B, Vol. 22, (5, Supplement 1), pp. 330-336, Oct., 2010.

Zhang, D.Z. and Prosperetti, A., "Averaged Equations for Inviscid Disperse Two-Phase Flow," Journal of Fluid Mechanics, Vol. 267, pp 185, 1994a.

Zhang, D.Z. and Prosperetti, A., "Ensemble Phase-Averaged Equations for Bubbly Flows”, Physics of Fluids, Vol. 6, pp. 2956-2970, 1994 b. 